This paper has been accepted for publication in Cognitive Development. The final article will be available, upon publication, via its DOI: https://doi.org/10.1016/j.cogdev.2022.101166

\title{
Perceptual Dissimilarity, Cognitive and Linguistic Skills Predict Novel Word Retention, but Not Extension Skills in Down Syndrome
}

\author{
Armando Q. Angulo-Chavira ${ }^{1 *}$ \\ ORCID: 0000-0002-6756-6717 \\ Roberto A. Abreu-Mendoza ${ }^{2 *}$ \\ ORCID: 0000-0002-6841-0917 \\ Marco A. Flores-Coronado 3 \\ ORCID: 0000-0003-0780-7934 \\ Elsa M. Vargas-García ${ }^{4}$ \\ ORCID: 0000-0003-0513-7690 \\ Natalia Arias-Trejo ${ }^{1}$ \\ ORCID: 0000-0002-4169-1616
}

\begin{abstract}
${ }^{1}$ Laboratorio de Psicolingüística, Facultad de Psicología, Universidad Nacional Autónoma de México.
${ }^{2}$ Department of Psychology, Rutgers University-Newark

${ }^{3}$ Laboratorio de Robótica Cognitiva, Centro de Investigación en Ciencias, Universidad Autónoma del Estado de Morelos.

${ }^{4}$ Facultad de Lenguas y Letras, Universidad Autónoma de Querétaro.

*These authors contributed equally to this work.
\end{abstract}

\section{Corresponding author details:}

Natalia Arias-Trejo

Faculty of Psychology, National Autonomous University of Mexico, Av. Universidad 3004, Sótano, Edificio "C," Col. Copilco Universidad, Coyoacan 04510, Ciudad de México, México. Tel. +52 55 56222287. Email: nariast@unam.mx.

\section{Declarations of Interest: none}

Data availability: The data that support the findings of this study are available from the corresponding author upon reasonable request.

Author contribution: Angulo-Chavira: methodology, investigation, data curation, formal analysis, writing - Original Draft, Writing - Review \& Editing, visualization, supervision, project administration. Abreu-Mendoza: methodology, writing - Original Draft, Writing - Review \& Editing, visualization. Flores-Coronado: methodology, formal analysis, software, writing Original Draft, Writing - Review \& Editing, visualization. Vargas-García: methodology, investigation, writing - Original Draft. Arias-Trejo: conceptualization, resources, writing Original Draft, Writing - Review \& Editing, supervision, project administration, funding acquisition. 
Acknowledgments: We wish to thank participants, parents, teachers and caregivers. We are also grateful to the members of the Laboratorio de Psicolingüística at UNAM for their help in testing participants, particularly to Liliana Fernández, Fernanda García and Liliana Santiago. 


\section{Highlights}

- Children with Down syndrome (DS) use the shape of objects to extend new labels.

- Mental age positively predicts retention of new labels in children with and without DS.

- Verbal mental age positively predicts retention of new labels in children with and without DS.

- Perceptual similarity of objects in learning environments drives the retention of new labels. 


\begin{abstract}
While the influence of cognitive and linguistic capacities and the perceptual features of objects on word-learning skills in people with typical development (TD) are well understood, there is little evidence concerning these mechanisms in people with Down syndrome (DS). Using a preferential looking task, we examined the ability of 29 children with DS (mean mental age: 3.44 years) to identify familiar words, fast-map pseudowords to novel objects, retain word-object mappings, and extend these mappings to new objects of similar shape. We also contrasted their word-learning abilities to those of 26 two-to-five-year-olds with TD and examined how cognitive and linguistic skills and perceptual information influenced those abilities. Children with DS were found to have similar identification, fast-mapping, and extension skills as their peers with TD, but retained fewer word-object mappings. Greater retention skills are related to mental age, verbal scores, and greater perceptual differences between the target and surrounding objects. These results indicate that children with DS use the same word-learning mechanisms and are influenced by the same linguistic and perceptual processes as children with TD.
\end{abstract}

Keywords: Down syndrome, fast mapping, word extension, word learning. 
Infants with typical development (TD) learn words at a formidable pace: they have learned the names of more than 100 referents by their second year (Jackson-Maldonado et al., 1993). Although apparently effortless, this word learning capacity is supported by sophisticated learning mechanisms, such as fast-mapping (Carey \& Barlett, 1978) and syntactic and morphological bootstrapping (Jolly \& Plunkett, 2008); it is constrained by heuristics such as the mutual exclusivity principle (Markman, 1990; Nelson, 1988; Spiegel \& Halberda, 2011) and the shape bias (Landau et al., 1988; Smith, 1995); and it is also assisted by sociopragmatic cues, such as the gaze of adults (Houston-Price et al., 2006). Notably, children with Down syndrome (DS) understand a comparable or greater number of words during the first years of life than their peers with TD matched by mental age (Galeote et al., 2011); however, it is still not known whether, in spite of multiple domain-general impediments such as a deficit in short-term memory (Jarrold et al., 2009), they learn words in the same way. In fact, little is known about the mechanisms that support word learning in children with this syndrome. Only a handful of studies have examined the skills used to fast-map novel words in children with DS (Chapman et al., 1990; Sakhon et al., 2018). A smaller number of studies have investigated processes beyond word-referent pairing and have focused mostly on whether children with DS can retain novel word-object mappings (Sakhon et al., 2018). In this study, we examine not only the ability of children with DS and TD to map novel words to unknown objects and retain those mappings, but also their ability to extend words to new category members. Then, in a series of exploratory analyses, we investigate the cognitive, linguistic, and perceptual correlates of word learning.

\section{Word Learning in Typical Development and Down Syndrome}

\section{Fast mapping and retention skills}


To learn words, children must extract them from a continuous acoustic stream that varies across speakers and contexts. Once a word has been identified, children must map it to one referent from a potentially infinite number of candidates (Quine, 1960), consolidate the wordreferent mapping in long-term memory, and then extend the word to new category members, with a few exceptions, like proper names (Gleitman, 1990; Hartley et al., 2019). At 17 months of age, children with TD can identify the correct referents of pseudowords using the mutual exclusivity principle: that is, with the heuristic of "one word, one referent," they can map novel words to unfamiliar objects when the objects are paired with familiar ones (Carey \& Barlett, 1978; Halberda, 2003; Markman, 1990). Their retention skills, however, are not yet as good. At the age of 2, children with TD retain new word-referent pairings for less than five minutes, and they fail to extend them to new category members (Horst \& Samuelson, 2008). Retention, however, improves rapidly. At 30 months, children tested 1.25 to 3.25 minutes after exposure to the referents can map up to six novel words and retain two words (Spiegel \& Halberda, 2011), and 3-year-olds can remember one recently learned word for at least a month (Markson \& Bloom, 1997).

Research on language development in DS concurs that regardless of the delay in productive vocabulary and grammatical skills over the course of development, children with this syndrome have a relative strength in their receptive vocabulary skills (Abbeduto et al., 2007). Children with DS show word comprehension skills according to their vocabulary size and follow the same lexical knowledge pattern (i.e., better performance for nouns than for verbs and attributes) as children with TD (Checa et al., 2016). If nonverbal growth in mental age is considered, children with DS show greater growth in receptive vocabulary than their peers with TD (Galeote et al., 2011, Mason-Apps et al., 2020). 
These studies provide a comprehensive description of the vocabulary profile in DS; however, research has often overlooked the mechanisms of word learning. One outstanding question is whether children with DS employ the same mechanisms as those with TD. Some studies have demonstrated that children with DS can use fast-mapping skills to learn novel words. In their seminal paper, Chapman et al. (1990) asked children and adolescents with DS and 2- to 6-year-olds with TD to hide two familiar and two unknown objects. One of the unknown objects was only pointed at, while the other was labeled with a novel word. Participants with DS and TD showed comparable abilities to comprehend, produce, and remember the location where the labeled novel object was hidden, both immediately and one hour later. Since then, studies have reported that adolescents and young adults with DS can learn up to four pseudowords and retain them for at least an hour, like 2- to 5-year-olds with TD (Chapman et al., 2006), and that they can learn pseudowords even in challenging grammatical contexts (McDuffie et al., 2007).

This prior research suggests that children with DS can learn new words after short exposure and retain them. However, except for McDuffie et al. (2007), these previous studies introduce novel words in contexts with only one object: the unknown object (i.e., they examine explicit mapping). The opportunities to learn new words from such contexts are uncommon, as parents often introduce new words in highly complex environments with multiple competitors (Clerkin et al., 2017). More recently, Sakhon et al. (2018) examined whether word learning is supported by the mutual exclusivity principle in children with DS. In their study, 3- to 5-yearolds with TD, and adolescents and adults with DS, learned four pseudowords in one of two word learning conditions. In one of these, pseudowords were accompanied by only one unknown object (an explicit encoding condition), and in the other, words were presented along with a pair of objects: a familiar and an unknown one (a fast-mapping condition). Notably, they found that 
children with DS demonstrated fast-mapping skills comparable to those with TD. Word retention was then evaluated at three intervals: immediately, five minutes later, and a week later. On average, both groups of children retained two words regardless of the learning condition or interval. These results dovetail with previous findings from explicit mapping studies (Chapman et al., 2006) showing similar word-retention skills in Down syndrome as in typical development.

In the current study, children with DS learned pseudowords in a more common context, one that presented them with multiple objects and required them to use the mutual exclusivity principle. Pseudowords were presented along with three familiar and one unknown object, and the children had to retain the novel word-to-object mappings for at least five minutes. To have a more precise measure of their retention skills, we tested eight novel word-object mappings. We hypothesized that children with DS would retain the same number of pseudowords as children with TD, as in previous studies (Chapman et al., 2006; Sakhon et al., 2018).

\section{Extension skills}

In addition to mapping and retaining word-object relations, children must also flexibly extend the labels of newly acquired words to different examples from the same category. A key ability in word learning is the capacity to extend words based on shape information (Golinkoff et al., 1992; Samuelson \& Smith, 1999). Under tight, low working memory experimental conditions, this capacity can be observed at the age of 18 months (Arias-Trejo, 2010). However, according to some authors, children can only reliably extend words after their productive vocabulary has reached 100 to 150 words (Samuelson \& Smith, 1999) and is followed by a rapid increase in vocabulary size. Training and individual difference studies have found that teaching young children to focus on shape information boosts their word learning (Smith et al., 2002), and 
that children who are better at remembering recently-learned, shape-related words, but not those related to colors or materials, have larger vocabularies (Perry et al., 2016).

Unlike studies of fast-mapping and retention skills, research on extension skills in DS is scarce. To the best of our knowledge, only Mervis and Bertrand (1995) have examined the ability of children with DS to extend newly acquired words. In their study, only nine of the 22 children with DS showed this capacity. However, because children were required to retain all the pseudowords throughout a fast-mapping task to then be tested in an extension phase, it remains unknown whether these results were due to the memory demands of the task, or a lack of ability related to DS. More recently, Barrón-Martínez and Arias-Trejo (2020) found that in children and adolescents with DS, words can be stored based on the perceptual similarities of their referents. In this priming study, participants with DS and 3-year-olds with TD had great difficulty in finding the referent of a familiar word (e.g., pencil) after hearing one with a referent of similar shape (e.g., arrow), suggesting that children with DS are sensitive to shape information in their lexical identification. These findings hint at the possibility that children with DS would extend the labels of referents under less demanding experimental conditions.

In the current study, we adapted the design of Rujas et al. (2019) to increase the likelihood of finding extension skills in children with DS. Their task assessed word learning across three conditions. In the first (identification phase), children had to identify the referent of a familiar word among three known objects and an unknown one; in the second (disambiguation phase), they saw the same objects as in the previous condition but had to point to the unknown object by mapping a pseudoword to it; in the third (extension phase), they saw two known objects and two unknown ones, one of the latter being the previously presented unknown object named with the pseudoword, but in a different color. We consider that this design reduces the 
load on working memory, as children have to generalize each pseudoword immediately after it is learned instead of at the end of the task. Thus, we predict that both children with DS and those with TD will successfully generalize the recently learned word-object mappings under these experimental conditions.

\section{Links Between Linguistic and Cognitive Skills and Word Learning}

Word learning skills do not develop in isolation; changes in cognitive and linguistic skills accompany them. In children with TD, individual differences in disambiguation but not identification skills are related to chronological age among 14- to 18-month-olds (Halberda, 2003). Greater disambiguation skills are also related to greater productive vocabulary and retention skills among those aged 18 to 30 months (Bion et al., 2013). In children with DS, word learning skills show different relationships than in those with TD. Comprehension of fastmapped words is related to measures of verbal mental age, while in those with TD, it is related to measures of syntactic complexity (Chapman et al., 2006). Children with DS do not benefit from hearing recently learned words multiple times (Chapman et al., 2006), while children with TD do (Lewis et al., 2020). Moreover, while the production of recently acquired words is strongly related to syntactic complexity in children with TD, it is related both to measures of syntactic complexity and of mental age in children with DS (Bird et al., 2004). Also, in children with DS, new word associations are independent of impairment in verbal working memory (Mosse \& Jarrold, 2011). However, there are also some commonalities: production of recently learned words is associated with larger expressive vocabularies in children with DS and TD (Bird et al., 2000).

Taken together, these findings suggest that while fast mapping skills in children with TD are mostly related to syntactic complexity (e.g., use of morphemes), in those with DS they are 
related both to measures of mental age and to syntactic complexity. However, the question remains as to whether word learning mechanisms beyond fast mapping are related to the same skills. Here we examine the relationships of both linguistic and non-linguistic abilities to extension and retention skills in children with DS and TD.

\section{Contextual Cues, Attention, and Word Learning}

Multiple lines of research have shown that word learning is influenced by the perceived context in which a new word is learned, in particular by the physical characteristics of the referent and the objects that surround it (Horst et al., 2010, 2020; Horst \& Samuelson, 2008; Houston-Price et al., 2006; Smith et al., 2010; Yu \& Smith, 2012). For example, children's word learning skills are enhanced when they encounter fewer competitors and when those competitors are familiar (Horst et al., 2010). Moreover, similarities between objects help children to note relevant information during naming. Recently, Horst et al. (2020) found that 30-month-old children and the iCub robot showed greater extension skills when an unknown object and two familiar objects had the same color than when the objects had different colors. According to the authors, controlling object color released attentional resources, which in turn allowed children to focus on the relevant feature of shape. However, there is also indirect evidence that similarities in the shape of objects may hinder word learning. Two-year-olds with TD have been found to erroneously overextend the label of familiar words (e.g., apple) to unknown objects with a similar shape (e.g., pomegranate; Gelman et al., 1998), and the word comprehension both of children with TD and of those with DS plummets after hearing a word with a similar perceptual referent (Barrón-Martínez \& Arias-Trejo, 2020).

In the current study, we investigate how visual features of objects affect word learning abilities, particularly extension and retention in children with and without DS. Using the 
Kullback-Leibler divergence (Kullback \& Leibler, 1951), a measure from information theory, we investigate how similarities in the perceptual features of objects affects word learning. If perceptual dissimilarity hinders word learning, word retention should be particularly challenging when objects are more dissimilar. If, however, dissimilarity facilitates word learning, we should observe the opposite pattern.

\section{The Current Study}

The aim of the current study was to comprehensively examine the word learning skills of children with DS and contrast them to those of children with TD matched by mental age. Using a preferential looking task, which provides fine-grained measures to examine the integration of linguistic and visual information (Huettig et al., 2011), we first assessed children's ability to identify familiar words (identification phase), fast-map pseudowords to novel objects (disambiguation phase), and extend these word-object mappings to novel objects of similar shape but different color (extension phase). Then, using an interactive task, we examined children's ability to retain these mappings after a five-minute delay (retention task). We hypothesized that children with DS and TD would show comparable word learning skills in the three phases of the preferential looking task and the retention task. We then leveraged the variability in children's performance in the preferential looking and retention tasks and in the perceptual features of our visual stimuli to explore the karyotype and linguistic, cognitive, and perceptual factors contributing to children's individual differences in word learning ability. Children with DS are a heterogeneous population: they can be born with regular trisomy or mosaicism, different genotypes that influence development of the physical, cognitive, behavioral, and linguistic phenotypes. We examined how these different genotypes and cognitive profiles of children with DS relate to their extension and retention skills. We then investigated whether verbal and 
nonverbal skills had different contributions for children with DS and TD. Consistent with prior research on retention skills, we hypothesized that linguistic and non-linguistic abilities would be related to both extension and retention skills in children with DS; for children with TD, the only linguistic ability would be related to these skills. Finally, we investigated how the perceptual features of objects (color and shape) hinder or facilitate word learning. We hypothesized that these features would affect both abilities: extension and retention; however, given the exploratory nature of this analysis, there is no clear evidence to support the directionality of such a relationship.

\section{Method}

\section{Participants}

Families of children with DS were invited to take part in the study through advertising in social media, by approaching parents at a specialized school for children with DS, and through word of mouth among parents of children with this syndrome. Families of TD children were first approached at a children's museum or through advertising in social media and were invited to participate if they had a child of similar mental age (MA) as children with DS. The final sample consisted of 55 monolingual Spanish-speaking children: 29 children with DS (19 boys), with a mean chronological age (CA) of 8.78 years $(S D=1.94$; range: $6.00-12.91)$ and a mean MA of

3.44 years $(S D=0.90$; range: $2.50-5.88)$; and 26 children with $\mathrm{TD}(18$ boys) with a mean CA of 3.02 years $(S D=0.56$; range: $2.50-4.83)$ and a mean MA of 3.42 years $(S D=0.86$; range: 2.50 5.83). There were no differences in gender between the children with DS and those with TD, $\mathrm{X}^{2}(1)=0.08, p=0.76$. We recruited participants with DS with a CA under 13 years and an MA of at least 2.5 years. Although there were differences in CA between groups, $Z(54)=6.36, p<$ 0.001 , there were no differences in MA, $Z(54)=0.16, p=0.87$. The group of 29 participants 
with DS included 20 children with regular trisomy 21, four with partial trisomy 21 (mosaicism), and five whose parents did not know their specific karyotype. Participants had normal or corrected-to-normal vision and an audiometry test found no severe hearing problems. Table 1 presents the demographics of the final sample.

Table 1

Demographic characteristics of the sample.

\begin{tabular}{lccc}
\hline & DS group & TD group & \multirow{2}{*}{$p$-value } \\
\cline { 2 - 3 } & $M(S D)$ & $M(S D)$ & \\
\hline Chronological age & $8.781(1.949)$ & $3.029(0.567)$ & $<0.001^{1}$ \\
Male/Female & $19 / 10$ & $18 / 8$ & $0.769^{2}$ \\
Mental age & $3.440(0.902)$ & $3.426(0.860)$ & $0.873^{1}$ \\
Caregiver education & $14.758(4.222)$ & $16.840(2.321)$ & $0.037^{1}$ \\
Understands* & $174.827(147.476)$ & $86.652(97.153)$ & $0.097^{1}$ \\
Understands/Says* & $283.241(222.750)$ & $467.956(144.955)$ & $0.006^{1}$ \\
Understands/Gestures* & $83.758(107.665)$ & $8.391(24.171)$ & $<0.001^{1}$ \\
\hline
\end{tabular}

Note: ${ }^{1} p$-values obtained with Mann-Whitney $U$-test. ${ }^{2} p$-values obtained with chi-square test. ${ }^{*}$ Scores from the communicative development inventory adapted for Down syndrome.

The initial sample consisted of 67 participants (38 with DS and 29 with TD). Twelve children were excluded, either because they did not perform a minimum number of usable trials in the experimental task ( 9 with DS and 2 with TD) or because they refused to continue with the preferential looking task ( 1 with TD). The sensitivity power analysis $(\alpha=0.05, \beta=0.80)$ performed in $\mathrm{g}^{*}$ power (Faul et al., 2009) showed that the final sample ( $n=55$, DS: $n=29$, TD: $n$ $=26$ ) allowed for the detection of medium within-subject differences (Cohen's $\delta=0.53$ ), and medium to large between-subject differences (Cohen's $\delta=0.77$ ), using a post-hoc $t$-test. Notably, the current sample size was larger than that of previous eye-tracking studies that reliably identified mapping and word retention skills in children with DS (Sakhon et al., 2018). However, this sample size was planned only for examining group differences between children with DS 
and TD and not for the exploratory analyses examining individual differences among them (see Results).

All participants were tested in (Blind region) Spanish, their caregivers gave written informed consent, and children gave oral assent for their participation. The study was approved by the blinded institutional research ethics committee (Approval No. blinded) and was in accordance with the principles of the Helsinki Declaration.

\section{Cognitive Assessments}

\section{Mental Age}

Mental age was determined using an abbreviated version of the Wechsler Preschool and Primary Scale of Intelligence (WPPSI-III; Wechsler, 2011) that includes the Receptive Vocabulary, Picture Naming, Block Design, and Object Assembly subtests. This abbreviated version has high reliability $(r=0.94)$, and validity $(s=0.78)$ scores, as compared with the complete test (Sattler, 2010).

In the Receptive Vocabulary subtest, children look at a group of four images and point to the one named by the experimenter. The subtest consists of 38 items which increase in difficulty as the test progresses. It ends when children have five consecutive errors or when they answer all items. Children are scored one point for each correct item.

In the Picture Naming subtest, children are shown one image at a time and are asked to name them. This subtest includes 30 pictures, increasing in difficulty as the subtest progresses. This test ends when children name all the images correctly or when they have five consecutive errors. Children are scored one point for every correctly named image without a penalty for mistakes. 
In the Block Design subtest, children are presented with models of blocks and are asked to recreate the design within a specific time limit. The test includes 20 models of increasing complexity. Children receive one point for each correctly constructed model plus a time bonus if they finish before the time limit. This test is complete when children have built all the models correctly or have three consecutive errors.

In the Object Assembly subtest, children are presented with 14 puzzles, one at a time. They receive one-half to one point for each correctly assembled piece, for a total of up to five points. The complexity increases as the test progresses with an increase in the number of pieces per puzzle. This test ends when children complete all the puzzles or when they score zero on three consecutive puzzles.

\section{Communicative Development Inventory}

As a part of the evaluation protocol, we administered the Communicative Development Inventory (CDI), a parent reporting instrument adapted for children with DS (Galeote et al., 2006). The inventory consists of a list of 651 early acquisition words where parents indicate whether children a) understand but do not produce a word, b) understand and produce a word orally, or c) understand and produce a word gesturally. The experimenter explained to parents the difference between comprehension and expression. Since parents are asked to indicate only one of the three options, the total vocabulary is the number of words marked as understood by the child regardless of their ability to express it. Here, we only report the descriptive results of the CDI because most of the children with TD and a few of those with DS showed a ceiling effect.

\section{Preferential Looking and Retention Tasks}

\section{Auditory Stimuli}


The auditory stimuli included eight familiar words and eight pseudowords (see Table 2). To ensure that children with DS would know the familiar words, we selected common concrete nouns from the DS-CDI (Galeote et al., 2006) with penult stress, given the high frequency of such words in Spanish (Quilis, 1983). Using the Wordbank Database for blinded Spanish (Frank et al., 2017; Jackson-Maldonado et al., 2003), we used the proportion of 30-month-old children with TD who produced each of the words. A Wordbank production score of one means that all children of a certain age produce a word; we obtained a mean score of $0.88(S D=0.07)$, indicating a strong likelihood that the participants in our study would know the words. To form the eight Spanish-like pseudowords, we followed the phonotactic rules of blinded Spanish, combining two high-frequency CV syllables with penult stress (Justicia, 1995), and verified that they did not appear in a Spanish dictionary (Real Academia Española, 2018).

All auditory stimuli, eight words and eight pseudowords, were recorded with a Shure MV51 microphone in a noise-proof booth by a female native Spanish-speaker using childdirected speech (Fernald, 1985). The mean duration of the auditory stimuli was $623.25 \mathrm{~ms}(S D=$ 63.55). Using Adobe Audition software, all stimuli were normalized to $-1.5 \mathrm{~dB}$, cleaned for background noise, and verified to have the acoustic characteristics of motherese.

\section{Table 2}

Experimental auditory stimuli.

\begin{tabular}{cc}
\hline Familiar word & Pseudoword \\
\hline árbol [tree] $(0.74)$ & du-fo \\
coche [car] $(0.95)$ & di-so \\
gato $[$ cat] $(0.92)$ & li-fo \\
globo $[$ balloon] $(0.84)$ & le-so \\
mano [hand] $(0.92)$ & gu-ro \\
mesa [table] $(0.92)$ & ga-fo
\end{tabular}


puerta [door] (0.84) ma-bo

zapato [shoe] $(0.89) \quad$ me-do

Note. Word familiarity, the proportion of 30-monthold children who can produce the word, is shown in parentheses (Frank et al., 2017; Jackson-Maldonado et al., 2003).

\section{Visual Stimuli}

The visual stimuli for the pseudowords were 24 novel objects (eight in two colors) selected from the Novel Objects Picture Database of the NOUN Database (Horst \& Hout, 2016). Objects for target novel words were selected to ensure they were distinct from the novel-object distractors presented in the extension phase and retention task based on the multidimensional scale distance. The overall mean distance in the Horst and Hout (2016) dataset was 0.85, and values ranged from 0.16 to 1.36 , where smaller values indicate a more similar pair of objects. For this study, the mean distance between a target image and the corresponding novel distractors was $0.87(S D=0.28)$. For the familiar words, visual stimuli were chosen from the concrete noun image database of the Psycholinguistics Laboratory at UNAM (https://osf.io/fyx8r/). Both familiar and novel objects were placed in a gray frame ( $800 \times 600$ pixels). Then, visual displays for the preferential looking tasks were constructed by placing four objects equidistantly in a 2 × 2 arrangement. Finally, for each trial, visual displays and their corresponding audios were combined in Flash-created videos.

For the retention task, images of objects were printed in high resolution on $140 \times 107.5$ mm cards and attached to a $340 \times 245 \mathrm{~mm}$ MDF board, with left, center, and right margins of 30 $\mathrm{mm}$ and top, middle, and bottom margins of $20 \mathrm{~mm}$.

\section{Designs}

Preferential Looking Task. To examine children's ability to identify familiar words and fast-map and extend pseudowords, participants performed a preferential looking task. Children 
first completed a five-point calibration procedure. They were then asked to look at the named object in a $2 \times 2$ object arrangement. They completed eight three-phase sets (identification, disambiguation, and extension), a total of 24 trials. In each trial, regardless of phase, the array of four objects was displayed for $6500 \mathrm{~ms}$, and the onset of the familiar word/pseudoword was at $3000 \mathrm{~ms}$ (Figure 1A). In the first phase, identification, children were asked to look at one of the familiar objects: they were shown an array of three familiar objects and one novel object, and heard a familiar word (Figure 1, lower left panel). This first phase not only allowed us to examine noun comprehension in general, but also to confirm that children understood the task. Immediately afterwards, in the disambiguation phase, they saw the same four objects as in the identification phase but heard a pseudoword rather than a familiar word (Figure 1, lower middle panel), requiring them to use the mutual exclusivity principle to disambiguate the pseudoword. Finally, in the extension phase, they were shown two familiar objects not previously seen, the labeled novel object in a different color, and an unseen novel object, with the same pseudoword auditory stimulus as in the previous phase (Figure 1, lower right panel). Here, they were required to extend the pseudoword to a novel object of the same shape as the one shown in the disambiguation phase, but in a different color.

The different colors for the labeled novel objects were taken from those proposed by Horst and Hout (2016) in their NOUN Database. The position of the objects was pseudorandomized across trials using Matlab, to ensure that the target was not in the same position in three sequential trials. Four pseudorandom sequences were created to present different object positions across participants. However, the objects were always paired with the same labels, and they were presented in different order. 


\section{Figure 1.}

Overview of the preferential looking and retention tasks

A. Preferential looking task
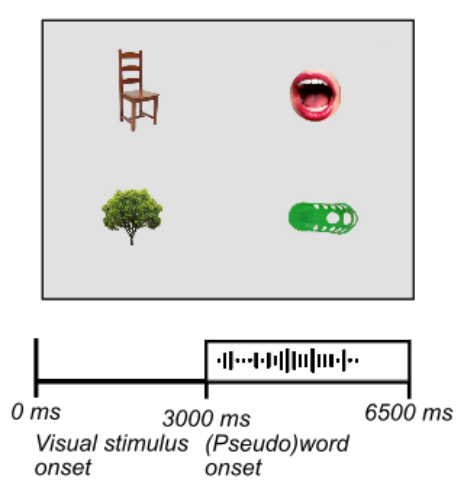

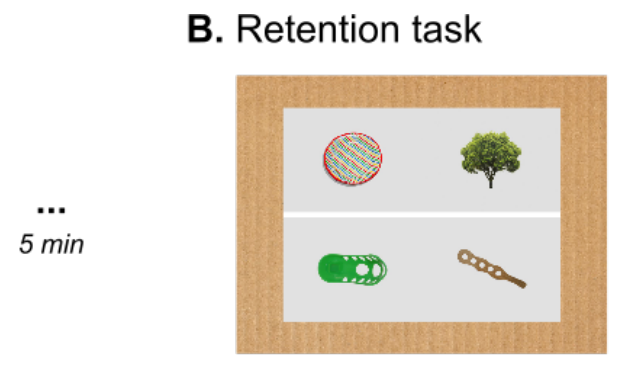

¿Cuál es el dufo? ¡Señala el dufo!
'Which one is the dufo? Point to the dufo'

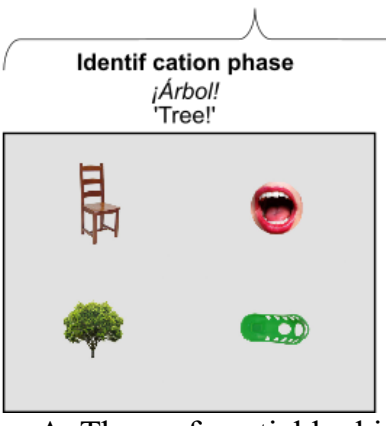

Disambiguation phase

Dufo!

Extension phase

Dufo!
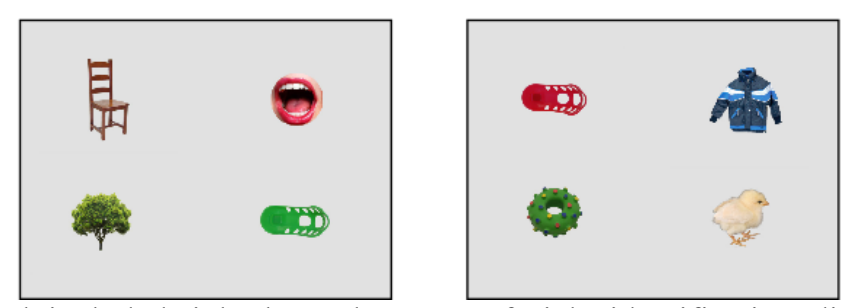

Notes. A. The preferential looking task included eight three-phase sets of trials: identification, disambiguation, and extension. B. The retention task five minutes after A.

Retention Task. To evaluate whether children could maintain the mapping between the pseudowords and novel objects, they were asked to perform a retention task five minutes after the preferential looking task (Figure 1B). In this task, they were asked to point to the labeled novel object (e.g., ¿Dónde está el mabo? Señala el mabo; "Where is the mabo? Point to the mabo"), presented in the same color as in the identification and disambiguation trials, together with images of a familiar object, an unseen familiar object, and an unseen novel object. There were a total of eight trials, equal in number to the pseudowords presented in the preferential looking task. The labeled novel objects were presented in the same order as in the preferential looking task to maintain similar times between learning and recalling.

\section{General Procedure}


First, parents provided informed consent and completed an interview about their children's health. Parents of children with DS were also asked whether they knew the genotype of their children and any comorbidities. Then, the children performed the MA assessment in a quiet room in the laboratory while the parents answered the CDI. When the children finished the MA assessment, the CDI was checked for errors or blank responses. Children were then taken to another room to perform the preferential looking task. Gaze data was collected using a Tobii Pro TX300 eye-tracker. In cases where children had to be seated on their parent's lap, parents were cautioned to remain silent. If children could sit by themselves, the parents stayed with the researcher, out of their children's sight. The children were asked to pay attention to the sounds and images on the screen and to remain quiet and still. After the preferential looking task, there was a five-minute break before the retention task. At the end of these tasks, families were compensated with a small gift for their participation in the study.

\section{Visual Stimulus Processing for Kullback-Leibler Divergence Estimates}

To quantify the perceptual dissimilarity of each labeled image in terms of shape and color, we used the Kullback-Leibler divergence (KLD, Kullback \& Leibler, 1951), a measure of the informational difference between two probability distributions, to compare the distributions of the color and shape image descriptors (Figure 2). This measure rests on the following theoretical assumptions: a) shape and color are the most relevant perceptual features in image perception (Holler et al., 2020); b) perception of object shape is constrained by the ways in which objects can be used to perform actions (Gibson, 1958); c) as in human perception, color does not vary with intensity of illumination (Swanson \& Cohen, 2003); d) perceptual 
dissimilarity between two object images is determined by differences in color and shape before perception; and e) shape and color have the same naive sensory relevance.

The visual stimuli were preprocessed in GIMP 2.1 to prepare them for feature extraction. To facilitate automatic picture extraction from the background, we changed the original gray background of each image to green. We then extracted shape and color descriptors using an adaptation of the algorithm in Goncalves et al. (2014). Shape features (e.g., squareness, circularity, and compactness) were extracted from a masked image version and from both halves of the objects. Color features were characterized as a hue-intensity histogram from each image HSV (hue, saturation, and value) color space. These processes produced two 17-dimension image descriptor vectors: a color descriptor vector and a shape descriptor vector. Shape and color features were extracted with Python 3.7 and Open CV 4.2.0.

The KLD values for each named-object/distractor-object pair were then calculated using SciPy (Virtanen et al., 2020). Larger KLD values indicate smaller overlap in the distributions of color and shape values between objects, and smaller values indicate a larger overlap. The values of the three pairs were then averaged (mean KLD) to obtain a single value for each of the arrays presented in the task, and this average was used as the independent variable for our analyses. The mean KLD value across all arrays was $2.49(S D=1.06)$.

Figure 2.

Overview of image processing and calculation of Kullback-Leibler divergence values.

A) Image Processing

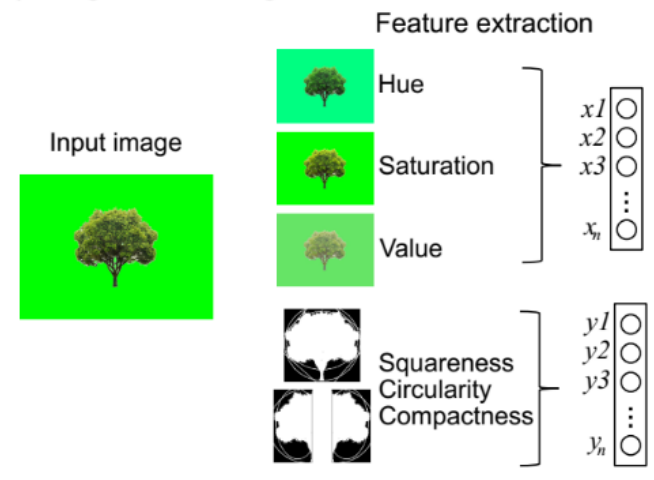

B) Kullback-Leibler Divergence estimation How dissimilar is [familiar object] from other objects in the trial?

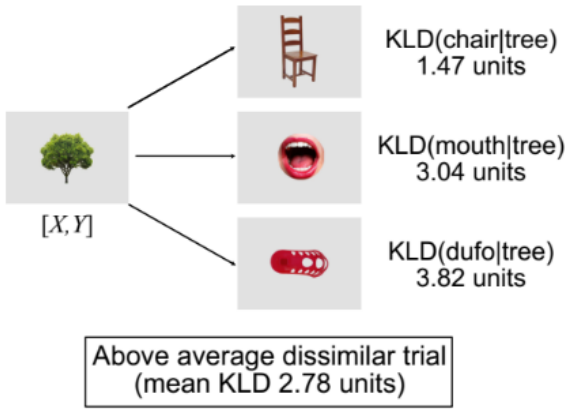


Note. A) The original gray background of each image was first changed to green to facilitate automatic object extraction. We then extracted color (hue, saturation, and value) and shape (squareness, circularity, and compactness) descriptors using an adaptation of the algorithm in Goncalves et al. (2014), which resulted in two 17-dimension image descriptor vectors: a color descriptor vector and a shape descriptor vector. B) To quantify the perceptual dissimilarity in shape and color of the named objects from the three distractor images, we calculated the KLD values for each named-object/distractor-object pair. KLD is an information-theory measure that estimates the informational distance between two distributions. Larger KLD values indicate that a pair of objects have a small overlap in their distributions of color and shape (e.g., the tree-dufo pair); smaller values indicate a larger overlap (e.g., the table-lifo pair). Finally, we averaged the values of the three pairs (mean KLD) to obtain a single value for each of the arrays presented in the task, which was used as the independent variable for our analyses.

To examine whether mean KLD values were normally distributed and compare them across the different phases of the preferential looking task, we first normalized them to a scale from -.05 to .05 . We then performed a Shapiro-Wilk test, which showed that the KLD values were not normally distributed (all $p$-values $<0.001$ ), and a Kruskal-Wallis test, which revealed differences in KLD scores between experimental phases, $\mathrm{X}^{2}(2)=307.38, p<0.001$; identification: $M=-0.03, S D=0.188$; disambiguation: $M=-0.12, S D=0.215$; extension: $M=$ $0.15, S D=0.189$. The post-hoc Wilcoxon test revealed that all comparisons were significant (all $p$-values $<0.001)$. These results show that the perceptual dissimilarity varied across phases and that each phase presented different competing demands in the processing of perceptual information.

\section{Data Recording, Data Processing and Statistical Analysis}

Children's gaze was recorded with a Tobii Pro TX-300 eye-tracker placed $\sim 60 \mathrm{~cm}$ from their eyes. This eye-tracker records binocular gaze position with a sampling rate of $300 \mathrm{~Hz}$ and an average accuracy of $0.5^{\circ}$ visual angle. To analyze their performance in the preferential looking task, four areas of interest were defined according to the size and location of each picture. Two analysis windows were evaluated independently for each phase (identification, disambiguation, extension): a pre-naming window from 300 to $3000 \mathrm{~ms}$ and a post-naming window from 3300 to $6000 \mathrm{~ms}$ after the trial onset. The $300 \mathrm{~ms}$ at the beginning of each window considers the time it 
takes the gaze to respond to stimuli (Canfield et al., 1997). Our main indicator of a successful performance is the increase in fixation time from the pre-naming (baseline) to the post-naming window. This indicator is a standard comparison in preferential looking tasks: it confirms that the fixations are driven by the auditory word, and not by visual saliency (Delle Luche et al., 2015).

Trials in which participants did not look for at least $300 \mathrm{~ms}$ in the pre-naming window and $300 \mathrm{~ms}$ in the post-naming window were excluded. After these exclusions, participants with fewer than four trials each phase were excluded (DS: $n=9$; TD: $n=2$ ). Of the remaining 55 participants (DS: $n=29$; TD: $n=26), 1217(92.19 \%)$ of the 1320 trials (55 participants $\times 3$ phases $\times 8$ trials) originally presented were analyzed: 414 for the identification phase (DS: $M=7.46, S D=0.89$; and TD: $M=7.60, S D=0.64$ per subject), 400 for the disambiguation phase (DS: $M=7.10, S D=1.29$; and TD: $M=7.48, S D=0.65$ per subject), and 403 for the extension phase (DS: $M=7.26, S D=$ 1.01; and TD: $M=7.40, S D=0.81$ per subject). The dependent variable for the analyses was the proportion of target looking (PTL), which was calculated independently for each phase as $P T L=$ $\frac{T}{\sum_{i=1}^{N} C_{i}}$, where $T$ is the total time of fixation towards the target images, $N$ is the total number of images presented including the target, and $C$ is the total fixation time on the $i$ th image. Analyses of Children's Performance on the Preferential Looking and Retention Tasks

To compare the word learning skills of children with DS and TD, we performed a 2 × 3 x 2 mixed ANOVA with Group (DS and TD) as between-subject factor, Phase (identification, disambiguation, and extension) and Window (pre-naming and post-naming) as within-subject factors, and the PTL scores as the dependent variable. Post-hoc analyses were performed using independent or paired $t$-tests where necessary. Retention skills between groups were compared using an independent $t$-test with the proportion of correct responses as a dependent variable. Exploratory Analyses Examining Individual Differences in Retention and Extension Skills 
The linguistic, cognitive, and perceptual factors contributing to extension and retention skills were explored using mixed effect models (see Supplementary Material 2 for disambiguation analyses). To perform models, the effect of target naming was computed as the difference between the PTLs in the post-naming and pre-naming windows in the extension phase, resulting in a new variable called $\Delta$ extension $(\Delta \mathrm{EXT})$. The continuous variables were normalized to a scale from -0.5 to 0.5 to improve the convergence of the models, and the nominal variables were dummy coded as factors in $\mathrm{R}$.

Extension skills were explored through linear mixed effect models (LMM) using $\Delta \mathrm{EXT}$ as a dependent variable; these models were performed with the lmer function from the lme4 packages (Bates et al., 2015) in R 3.6 (R Core Team, 2019). Retention skills were analyzed using a binomial logistic mixed effect model (BMM) using the binary variable of correct vs. incorrect response in the retention task; these models were built using the glmer function from the lme4 packages (Bates et al., 2015) in R 3.6 (R Core Team, 2019).

The analysis strategy consisted of a model comparison (Chambers, 1992) using the anova function. Thus, a reference model was created for both dependent measures, including only the intercept as a predictor and the subjects as a random factor. Potential fixed factors were added hierarchically to the model, one by one. The addition of the fixed factor considered the main effects and the interaction among predictors. The random factors were then introduced hierarchically, using the best-fitted model from the previous step to keep the complex structure that converged (Barr, 2008). The intercepts of subjects, trials, sequences, and items were included as random factors (Baayen et al., 2008). The trial factor was each picture array in the preferential looking task $(n=24)$. The sequence factor was each of the four possible pseudorandom orders of the pictures in the array (see Preferential Looking Task section). The 
item factor was each of the eight trained novel objects. After the inclusion of fixed and random factors, the best fitted model was explored, and it was assumed that the variables that did not improve the model were not relevant to explanation of the dependent variable.

A total of three model comparisons were performed with extension and retention skills. The first explores individual differences in the genotypes, chronological ages, and mental ages. As karyotype describes only children with DS, and chronological and mental ages are highly correlated among those with TD, our first exploratory analysis focused only on those with DS. The fixed factors and their interactions were introduced hierarchically in the following order: mental age, chronological age, and karyotype. Karyotype was introduced last because there were only four participants with mosaicism, and the karyotype of several participants was unspecified.

The second model comparison explored different contributions of verbal and nonverbal skills for extension and retention. This comparison included both groups, since individuals with DS performed better at nonverbal than at verbal tasks (Silverman, 2007). The mental age scores were split into two components: verbal (receptive vocabulary and picture naming) and nonverbal (block design and puzzles). Although people with DS usually have better receptive than expressive skills, the measures were averaged to avoid collinearity, given their high degree of correlation $(r=.62, p<0.001)$. The fixed factors and their interactions were introduced hierarchically in the following order: verbal skills, non-verbal skills, and group.

Finally, a third model comparison explored the effect of the perceptual learning environment in both groups using the mean KLD as the indicator of this process. The fixed factors and their interactions were introduced hierarchically in the following order: mean KLD, phase (for retention analysis only), and group.

\section{Results}




\section{Children with DS and TD Have Similar Identification, Disambiguation, and Extension Skills}

A Shapiro-Wilk test showed that the PTL scores were normally distributed, with all $p$ values $>0.09$, and the Levene test showed that the variances were homogeneous between groups, with all $p$-values $>0.24$.

The ANOVA yielded significant main effects of Phase, $F(2,106)=19.55, p<0.001, \eta_{\mathrm{p}}{ }^{2}$ $=0.27$, and Window, $F(1,23)=84.71, p<0.001, \eta_{\mathrm{p}}{ }^{2}=0.6$, but the main effect of Group was not significant, $F(1,53)=1.73, p=0.19, \eta_{\mathrm{p}}{ }^{2}=0.03$. Neither the interaction between Group and Window, $F(1,53)=0.61, p=0.80, \eta_{\mathrm{p}}^{2}=0.001$, nor the interaction among Group, Phase, and Window, $F(2,106)=0.16, p=0.84, \eta_{\mathrm{p}}{ }^{2}=0.003$, reached statistical significance. However, there was a significant interaction between Phase and Group, $F(2,106)=4.60, p=0.01, \eta_{\mathrm{p}}{ }^{2}=0.08$, which showed that children with DS looked less at the target in the extension phase than those with TD, $t(53)=2.94, p=0.005, \delta=0.79$, regardless of the Window factor; this difference was not found in the identification phase, $t(53)=0.69, p=0.85, \delta=0.18$, or the disambiguation phase, $t(53)=0.18, p=0.85, \delta=0.05$.

The two significant main effects were qualified by an interaction between Phase and Window, $F(2,106)=24.30, p<0.001, \eta_{\mathrm{p}}{ }^{2}=0.31$, indicating that the increase in looking time from the pre- to the post-naming window varied through all three phases, but was similar between groups (Figure 3). Post-hoc analyses found that participants in both groups looked more in the post-naming than in the pre-naming window across all phases, identification: $t(54)=9.74$, $p<0.001, \delta=1.31$; disambiguation: $t(54)=3.18, p=0.002, \delta=0.42$; and extension: $t(54)=$ $2.28, p=0.002, \delta=0.44$. They also looked more in the post-naming window of the identification phase than in the disambiguation phase, $t(54)=6.46, p<0.001, \delta=1.09$, and the extension 
phase, $t(54)=7.39, p<0.001, \delta=1.60$. Notably, there were no significant differences between the pre-window looking times of the three phases (all $p$-values $<0.05$ ).

Table 3

Central tendency measures of the PTL

\begin{tabular}{llcc}
\hline Phase & Group & $\begin{array}{c}\text { Pre-naming } \\
\text { M (SD) }\end{array}$ & $\begin{array}{c}\text { Post-naming } \\
\text { M (SD) }\end{array}$ \\
\hline \multirow{2}{*}{ IDE } & TD & $0.23(0.09)$ & $0.45(0.1)$ \\
& DS & $0.25(0.09)$ & $0.45(0.13)$ \\
\multirow{2}{*}{ DIS } & TD & $0.25(0.08)$ & $0.31(0.11)$ \\
& DS & $0.25(0.09)$ & $0.31(0.13)$ \\
EXT & TD & $0.27(0.08)$ & $0.32(0.12)$ \\
& DS & $0.21(0.09)$ & $0.26(0.1)$ \\
\hline
\end{tabular}

Overall, these results suggest that children from the two groups have comparable identification, disambiguation, and extension skills (Table 3). Sixty-five percent of the participants in the DS group and 65\% in the TD group increased their looking in the post-naming window of the extension phase. This implies that most participants can perform extensions using the shape of the new learned object; however, a few participants have problems extending new learned labels. 
Figure 3.

Performance on the preferential looking task in pre-naming and post-naming windows.

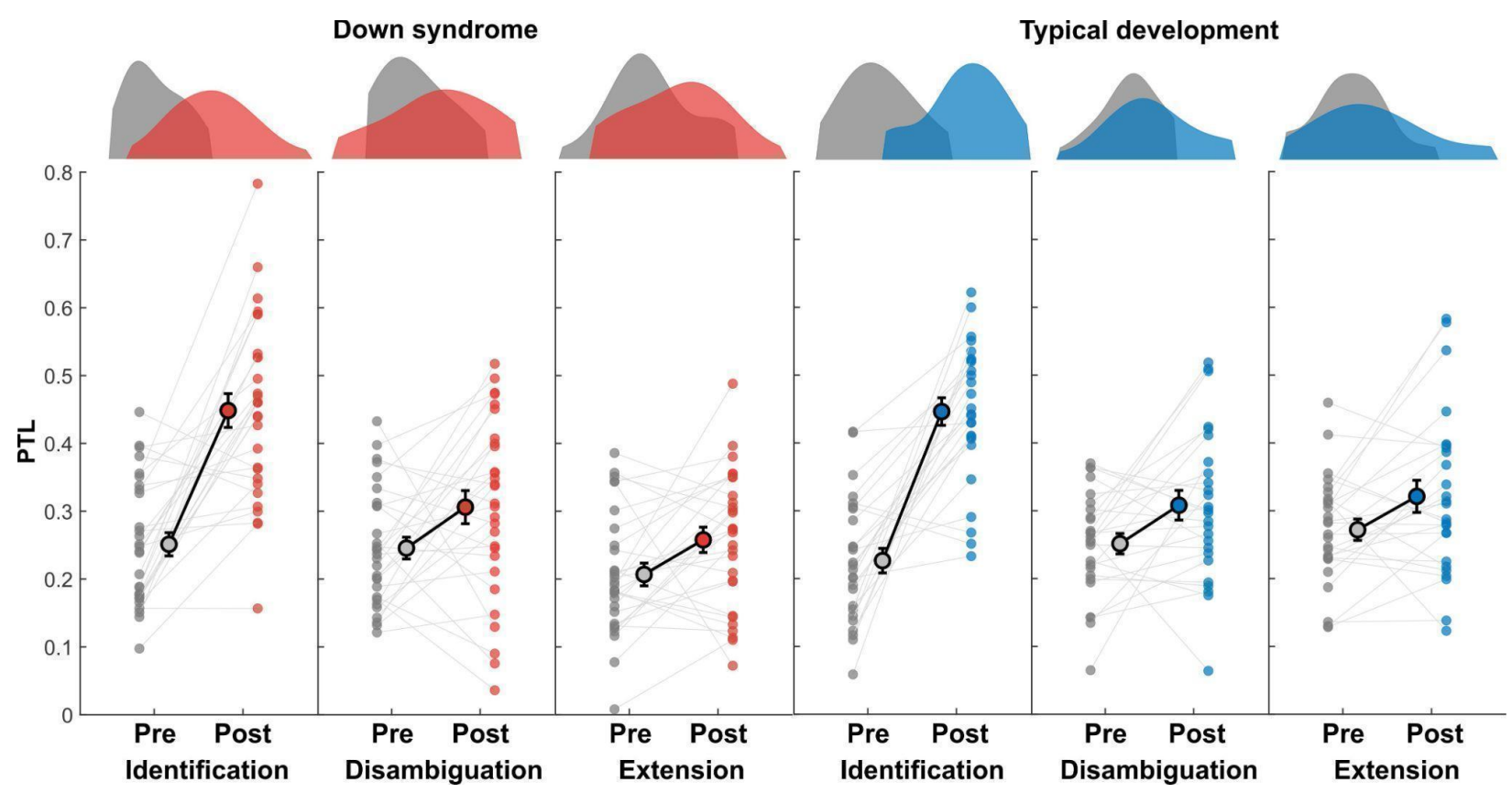

Notes: Density plots represent the marginal distributions of the proportion of target looking (PTL). Gray dots represent the pre-naming PTL scores, while red and blue dots represent the post-naming PTL scores of the children with DS and TD, respectively. Error bars indicate the mean and \pm 1 standard error.

\section{Children with DS Retain Fewer Novel Words than Those with TD}

Of the 55 participants in the sample, two participants from the TD group were not included in the following analyses, as they did not want to continue with the evaluation. A Shapiro-Wilk test showed that the proportion of correct responses (PCR) was normally distributed, with all $p$-values $>0.12$, and the Levene test showed that the variances were homogeneous between groups, with $p=0.07$.

These analyses indicated that children with DS remembered fewer items than those with $\mathrm{TD}, t(51)=2.11, p=0.03, \delta=0.58$ (Figure 4), meaning that children with DS remember fewer novel word-object mappings after a brief exposure $(M=3.13, S D=2.06$, range: $0-8)$ than children with TD $(M=4.22, S D=1.50$, range: $1-8)$. 


\section{Figure 4.}

Performance on the retention task.

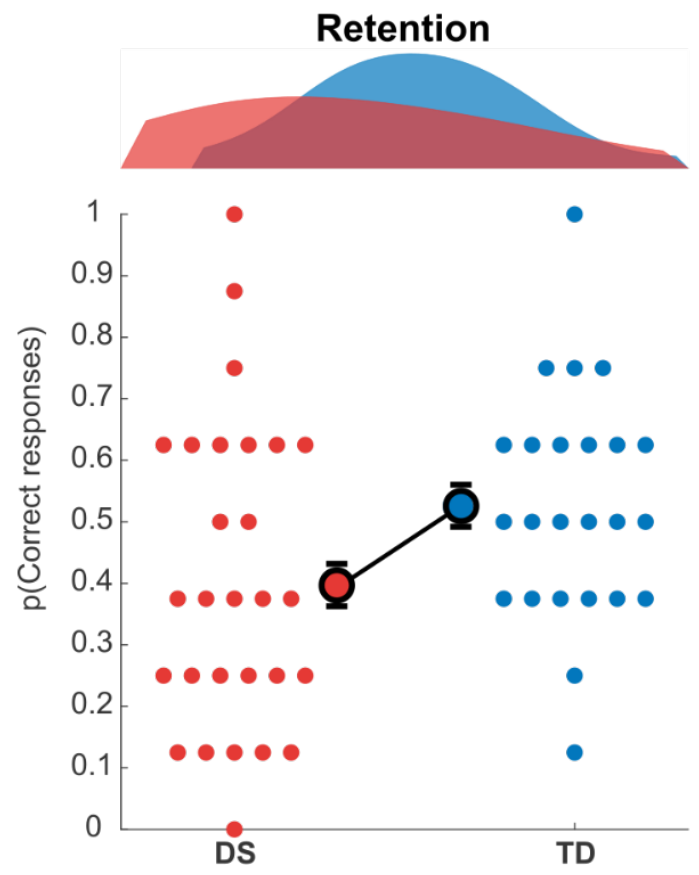

Notes: Density plots represent the marginal distributions of the proportion of correct responses (PCR). Red dots represent the proportion of words remembered by children with DS and blue dots those with TD. Error bars indicate the mean and \pm 1 standard error.

\section{Retention, but Not Extension, is Related to Cognitive Profiles of Children with DS}

The LMM model for extension skills showed that neither mental age, chronological age, nor karyotype increased the fit of the model (all $p$-values $>0.05$ ), indicating that they did not contribute to the extension skills in the DS group (see Table 4).

\section{Table 4}

Model comparison for individual differences of extension skills in children with DS.

\begin{tabular}{lcccccc}
\hline \multicolumn{1}{c}{ Models } & NP & AIC & Ln(L) & X $^{2}$ & df & p \\
\hline$\Delta \mathrm{PTL} \sim 1+(1 \mid$ Subj $)$ & 3 & -107.86 & 56.93 & & & \\
$\Delta \mathrm{PTL} \sim \mathrm{MA}+(1 \mid$ Subj $)$ & 4 & -106.56 & 57.281 & 0.703 & 1 & 0.402 \\
$\Delta \mathrm{PTL} \sim \mathrm{MA} * \mathrm{CA}+(1 \mid$ Subj $)$ & 6 & -103.09 & 57.547 & 0.532 & 2 & 0.767 \\
$\Delta \mathrm{PTL} \sim \mathrm{MA} * \mathrm{CA} * \mathrm{KT}+(1 \mid$ Subj $)$ & 14 & -92.991 & 60.496 & 5.898 & 8 & 0.659 \\
\end{tabular}


Notes. NP: number of parameters; AIC: Akaike information criterion; $\operatorname{Ln}(\mathrm{L})$ : log-likelihood; $\triangle \mathrm{PTL}$ : the difference of proportion of target looking (Post-naming - Pre-naming); MA: mental age; CA: chronological age; KT: karyotype; Subj: subject.

Table 5

Model comparisons for retention task in children with DS.

\begin{tabular}{|c|c|c|c|c|c|c|}
\hline Fixed factors & & & & & & \\
\hline Models & NP & AIC & $\operatorname{Ln}(L)$ & $\mathbf{X}^{2}$ & $d f$ & $p$ \\
\hline $\mathrm{CR} \sim 1+(1 \mid$ Subj $)$ & 2 & 751.569 & -373.78 & & & \\
\hline CR $\sim$ MA + (1 | Subj $)$ & 3 & 742.054 & -368.03 & 11.515 & 1 & 0.001 \\
\hline $\mathrm{CR} \sim \mathrm{MA} * \mathrm{CA}+(1 \mid \mathrm{Subj})$ & 5 & 741.421 & -365.71 & 4.633 & 2 & 0.099 \\
\hline $\mathrm{CR} \sim \mathrm{MA} * \mathrm{CA} * \mathrm{KT}+(1 \mid$ Subj $)$ & 13 & 748.455 & -361.23 & 8.965 & 8 & 0.345 \\
\hline \multicolumn{7}{|l|}{ Random factors } \\
\hline $\mathrm{CR} \sim \mathrm{MA}+(1 \mid$ Subj $)$ & 3 & 742.054 & -368.03 & & & \\
\hline CR $\sim$ MA + (1 | Subj $)+(1 \mid$ Item $)$ & 4 & 732.724 & -362.36 & 11.329 & 1 & 0.001 \\
\hline
\end{tabular}

Notes. NP: number of parameters; AIC: Akaike information criterion; $\operatorname{Ln}(\mathrm{L})$ : log-likelihood; CR: correct responses in the retention task; MA: mental age; CA: chronological age; KT: karyotype; Subj: subject; Item: remembered words. Bold values indicate significant effects.

By contrast, the BMM retention model showed that mental age increased the fit of the model $(p=0.001)$, but the chronological age and karyotype did not $(p>0.09)$, suggesting that neither chronological age nor karyotype explain the word retention in the DS group. However, this result should be interpreted with caution since the karyotypes are not well represented in this sample. The best model included mental age as a fixed factor and the intercepts of subject and trial as random factors (see Table 5), indicating that children with DS increased their retention in parallel with their mental age $(\beta=3.033, S E=0.642, z=4.726, p<0.001$; see Figure 5). Note that introducing the Group factor to the model does not improve the fit, $\mathrm{X}^{2}(2)=4.83, p=0.08$, suggesting that mental age predicts retention skills similarly in both groups (Figure 5). 
Figure 5.

Modulation of mental age on retention skills

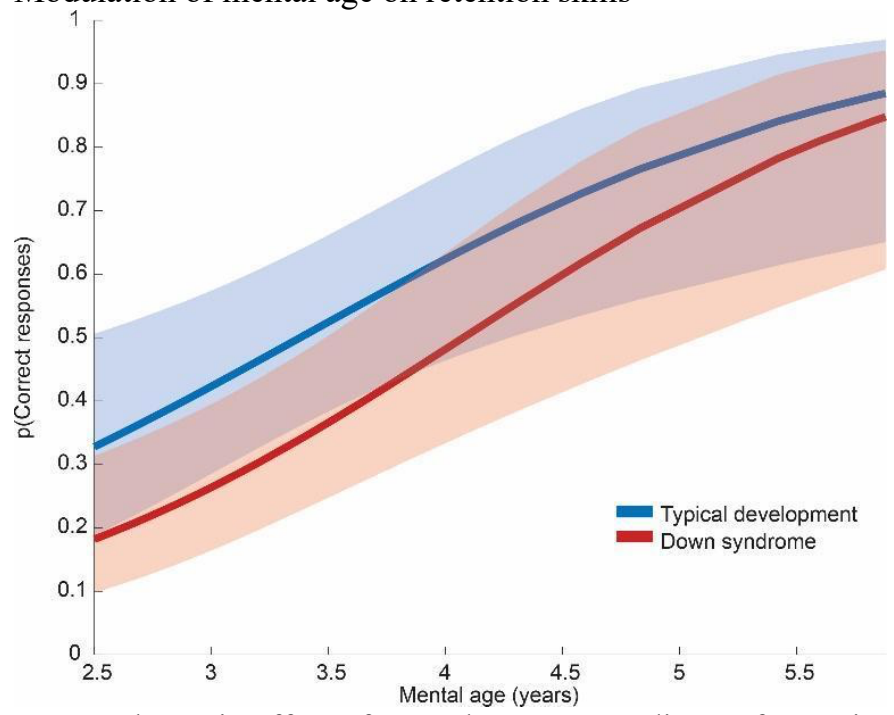

Notes. The main effect of mental age as a predictor of retention skills. The lines represent the predicted values for correct responses, and shaded areas indicate a 95\% confidence interval.

\section{Language Skills are Related to Retention Skills in Children with and without DS}

None of the fixed factors (verbal, nonverbal, group) increased the fit of the LMM model for extension skills (all $p$-values $>0.05$ ), indicating that extension was not explained by verbal or non-verbal variables, and those skills were not different between groups (see Table 6).

Table 6

Model comparison for verbal and nonverbal skills in the extension phase for both groups.

\begin{tabular}{|c|c|c|c|c|c|c|}
\hline Models & NP & AIC & $\mathbf{L n}(\mathbf{L})$ & $\mathbf{X}^{2}$ & $\begin{array}{l}d \\
f\end{array}$ & $p$ \\
\hline$\Delta \mathrm{PTL} \sim 1+(1 \mid$ Subj $)$ & 3 & -191.15 & 98.575 & & & \\
\hline$\Delta \mathrm{PTL} \sim$ Verbal $+(1 \mid$ Subj $)$ & 4 & -190.71 & 99.356 & 1.56 & 1 & $\begin{array}{c}0.21 \\
2\end{array}$ \\
\hline$\Delta \mathrm{PTL} \sim$ Verbal $*$ Nonverbal $+(1 \mid$ Subj $)$ & 6 & -188.28 & $\begin{array}{c}100.13 \\
7\end{array}$ & $\begin{array}{c}1.56 \\
3\end{array}$ & 2 & $\begin{array}{c}0.45 \\
8\end{array}$ \\
\hline$\Delta \mathrm{PTL} \sim$ Verbal $*$ Nonverbal $*$ Group $+(1 \mid$ Subj $)$ & 10 & -183.02 & $\begin{array}{c}101.51 \\
2\end{array}$ & $\begin{array}{c}2.74 \\
9\end{array}$ & 4 & $\begin{array}{c}0.60 \\
1\end{array}$ \\
\hline
\end{tabular}

Notes. NP: number of parameters; AIC: Akaike information criterion; $\operatorname{Ln}(\mathrm{L})$ : log-likelihood; $\triangle \mathrm{PTL}$ : the difference of proportion of target looking (post-naming/pre-naming); Verbal: average of verbal scores in receptive vocabulary and picture naming; Nonverbal: average of scores from cube design and puzzles; Subj: subject. 


\section{Figure 6.}

Modulation of verbal scores on retention skills.

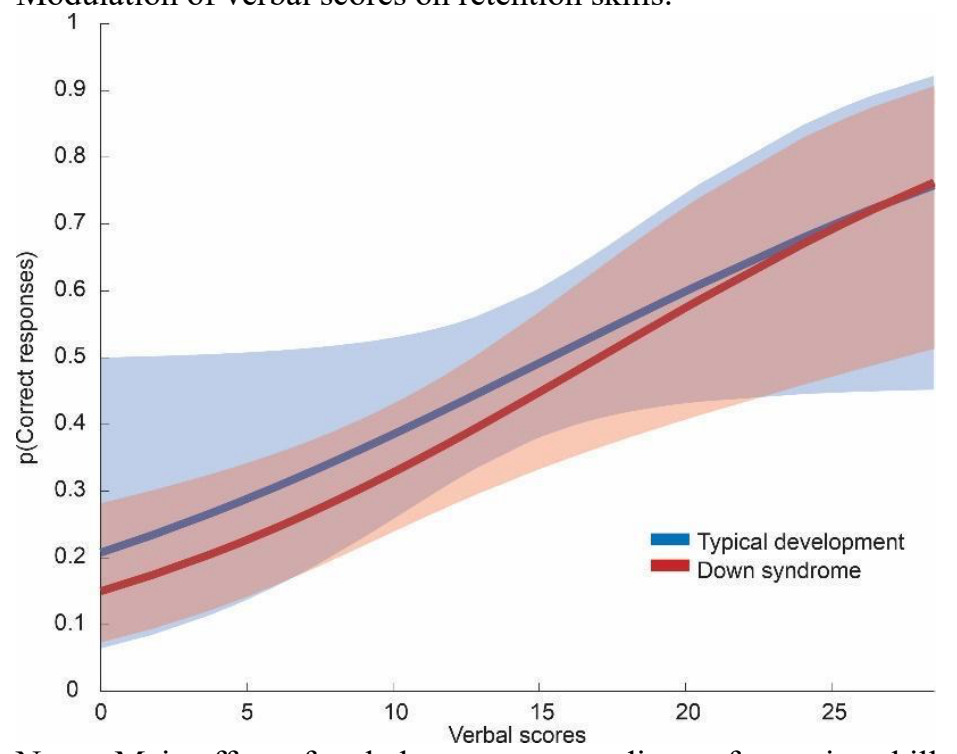

Notes. Main effect of verbal scores as a predictor of retention skills. The lines represent the predicted values for correct responses, and shaded areas indicate a 95\% confidence interval.

In contrast, in the retention skills, the BMM showed that verbal scores (SD: $M=11.70$, $S D=7.25$; TD: $M=14.72, S D=5.05)$ increased the fit of the intercept model $(p<0.001)$, but neither nonverbal scores (SD: $M=14.31, S D=7.34$; TD: $M=11.92, S D=5.91$ ) nor group did so $(p>0.29)$. The best model, which included verbal scores as a fixed factor and the subjects and items as a random factor (Table 7), revealed that participants with and without DS with greater vocabulary skills were the ones who retained more novel words $(\beta=3.033, S E=0.642, z=$ 4.726, $p<0.001$; Figure 6). These results suggest that word retention skills are related to receptive and expressive vocabulary, both in children with and without DS.

\section{Table 7}

Model comparison for verbal and nonverbal skills in the retention task for both groups.

\begin{tabular}{lcccccc}
\hline \multicolumn{1}{c}{ Fixed factors } \\
\multicolumn{1}{c}{ Models } & NP & AIC & Ln(L) & $\mathbf{X}^{2}$ & df & $\boldsymbol{p}$ \\
\hline $\mathrm{CR} \sim 1+(1 \mid$ Subj $)$ & 2 & 1504.05 & -750.02 & & & \\
$\mathbf{C R} \sim$ Verbal $+(\mathbf{1} \mid$ Subj $)$ & $\mathbf{3}$ & $\mathbf{1 4 9 0 . 4 9}$ & $\mathbf{- 7 4 2 . 2 5}$ & $\mathbf{1 5 . 5 5 6}$ & $\mathbf{1}$ & $<\mathbf{0 . 0 0 1}$ \\
$\mathrm{CR} \sim$ Verbal * Nonverbal $+(1 \mid$ Subj $)$ & 5 & 1492.03 & -741.02 & 2.457 & 2 & 0.293
\end{tabular}




\begin{tabular}{llrrrrr}
$\begin{array}{l}\text { CR } \sim \text { Group * Verbal * Nonverbal }+(1 \mid \\
\text { Subj })\end{array}$ & 9 & 1496.62 & -739.31 & 3.419 & 4 & 0.49 \\
\hline \multicolumn{7}{c}{ Random factors } \\
\hline CR $\sim$ Verbal $+(1 \mid$ Subj $)$ & 3 & 1490.49 & -742.25 & & & \\
CR $\sim$ Verbal $+(\mathbf{1} \mid$ Subj $)+(\mathbf{1} \mid$ Item $)$ & $\mathbf{4}$ & $\mathbf{1 4 4 1 . 2 9}$ & $\mathbf{- 7 1 6 . 6 5}$ & $\mathbf{5 1 . 1 9 9}$ & $\mathbf{1}$ & $<\mathbf{0 . 0 0 1}$ \\
\hline
\end{tabular}

Notes. NP: number of parameters; AIC: Akaike information criterion; $\operatorname{Ln}(\mathrm{L})$ : log-likelihood; CR: correct responses in the retention task; Verbal: average of verbal scores in receptive vocabulary and picture naming; NonVerbal: average of scores from cube design and puzzles; Subj: subject; Item: remembered the word. Bold values indicate significant effects.

\section{Perceptual Dissimilarity Affects Word Learning in Children with DS and TD}

The LMM model for extension skills revealed that neither the mean KLD nor Group increased the fit of the model (all $p$-values $>0.05$ ), indicating that these factors did not contribute to extension skills (see Table 8).

The retention skills BMM showed that $\operatorname{KLD}(p=0.02)$ and phase $(p=0.03)$ but not group $(p=0.061)$ increase the fit of the intercept model. The best model included KLD and phase as fixed factors and the subjects and trials as random factors (Table 9). The exploration of the best fitted model showed a significant main effect of $\operatorname{KLD}(p=0.007)$; this effect was qualified by an interaction between KLD and phase, revealing that the mean KLD in the identification phase was different from the mean KLD in the disambiguation phase $(p=0.01)$, but not in the extension phase $(p=0.21$; Table 10). To better understand this interaction between KLD and phase, we split the data and performed simpler models which included only the KLD factor for each phase. These analyses showed that mean KLD scores in the identification phase were a negative predictor of word retention $(\beta=-1.918, S E=0.646, z=-2.968, p=0.003$; Figure 7); however, the KLD in the disambiguation phase $(\beta=0.332, S E=0.576, z=0.577, p=0.546)$, and the extension phase $(\beta=$ $-0.815, S E=0.634, z=-1.287, p=0.198)$, was not significant. These results suggest that the dissimilarity of the familiar target in the identification phase influenced the word retention of novel objects similarly in both groups; a high degree of dissimilarity hindered word learning. 
Table 8

Model comparison for Kullback-Leibler divergence in the extension phase.

\begin{tabular}{lcccccc}
\multicolumn{1}{c}{ Models } & NP & AIC & Ln(L) & $\mathbf{X}^{2}$ & $\boldsymbol{d f}$ & $\boldsymbol{p}$ \\
\hline$\Delta \mathrm{PTL} \sim 1+(1 \mid$ Subj $)$ & 3 & -213.72 & 109.862 & & & \\
$\Delta \mathrm{PTL} \sim \mathrm{KLD}+(1 \mid$ Subj $)$ & 4 & -213.25 & 110.624 & 1.523 & 1 & 0.217 \\
$\Delta \mathrm{PTL} \sim \mathrm{KLD} *$ Group $+(1 \mid$ Subj $)$ & 6 & -209.3 & 110.648 & 0.05 & 2 & 0.975 \\
\hline
\end{tabular}

Notes. NP; number of parameters; AIC; Akaike information criterion; Ln(L); log-likelihood; $\Delta \mathrm{PTL}$; difference in proportion of target looking (post-naming/pre-naming); KLD; mean Kullback-Leibler divergence; Subj; subject.

Table 9

Model comparison for Kullback-Leibler divergence in retention task.

\begin{tabular}{|c|c|c|c|c|c|c|}
\hline \multicolumn{7}{|l|}{ Fixed factors } \\
\hline Models & NP & AIC & $\operatorname{Ln}(\mathbf{L})$ & $\mathbf{X}^{2}$ & $d f$ & $p$ \\
\hline $\mathrm{CR} \sim 1+(1 \mid \mathrm{Subj})$ & 2 & 1515.31 & -755.65 & & & \\
\hline $\mathbf{C R} \sim \mathbf{K L D}+(\mathbf{1} \mid \mathbf{S u b j})$ & 3 & 1512.87 & -753.44 & 4.433 & 1 & 0.035 \\
\hline CR $\sim$ KLD $*$ Phase $+(1 \mid$ Subj $)$ & 7 & 1510.11 & -748.06 & 10.764 & 4 & 0.029 \\
\hline $\mathrm{CR} \sim \mathrm{KLD} *$ Phase $*$ Group $+(1 \mid$ Subj $)$ & 13 & 1517.61 & -745.81 & 4.498 & 6 & 0.61 \\
\hline \multicolumn{7}{|l|}{ Random factors } \\
\hline $\mathrm{CR} \sim \mathrm{KLD} *$ Phase $+(1 \mid$ Subj $)$ & 7 & 1510.11 & -748.05 & & & \\
\hline CR $\sim$ KLD $*$ Phase $+(1 \mid$ Subj $)+(1 \mid$ Trial $)$ & 8 & 1500.68 & -742.34 & 11.426 & 1 & $<\mathbf{0 . 0 0 1}$ \\
\hline $\begin{array}{l}\mathrm{CR} \sim \mathrm{KLD} * \text { Phase }+(1 \mid \text { Subj })+(1 \mid \text { Trial })+ \\
(1 \mid \text { Seq })\end{array}$ & 9 & 1502.68 & -742.34 & 0 & 1 & 1 \\
\hline
\end{tabular}

Notes. NP: number of parameters; AIC: Akaike information criterion; Ln(L): log-likelihood; CR: correct responses in the retention task; KLD: mean Kullback-Leibler divergence; Phase (Identification, Disambiguation, Extension); Subj: subject; Item: remembered word; Trial: number of observed trials; Seq: presentation order of the image array. Bold values indicate significant effects. 
Table 10

Best fit model for perceptual exploration.

\begin{tabular}{|c|c|c|c|c|}
\hline Fixed effects & B & $S E$ & $Z$ & $p$ \\
\hline Intercept & -0.334 & 0.244 & -1.367 & 0.172 \\
\hline KLD & -2.013 & 0.745 & -2.703 & 0.007 \\
\hline PhaseDIS & 0.051 & 0.256 & 0.199 & 0.842 \\
\hline PhaseEXT & 0.127 & 0.274 & 0.463 & 0.643 \\
\hline KLD:PhaseDIS & 2.354 & 0.958 & 2.458 & 0.014 \\
\hline KLD:PhaseEXT & 1.246 & 1.004 & 1.241 & 0.214 \\
\hline
\end{tabular}

Notes. Formula: $\mathrm{CR} \sim \mathrm{KLD} *$ Phase $+(1 \mid$ Subj $)+(1 \mid$ Trial $)$. KLD: mean Kullback-Leibler divergence. Bold values indicate significant effects.

Figure 7.

Modulation of the perceptual environment in word learning.

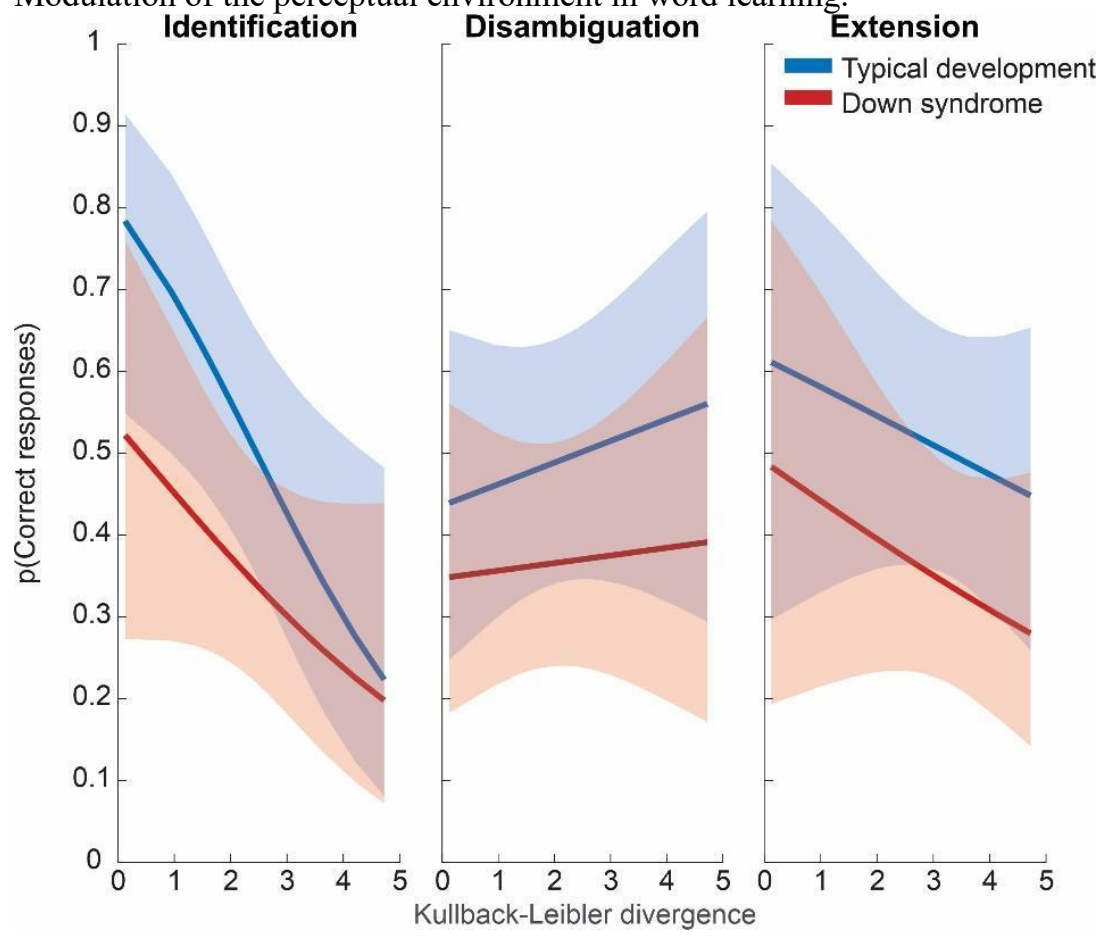

Notes. Model interaction between mean KLD scores (greater values equal greater dissimilarity) and word learning phase. The lines represent the predicted values for correct responses, and shaded areas indicate a $95 \%$ confidence interval.

\section{Discussion}

This study investigated the ability of children with Down syndrome (DS) to map new words to novel objects, retain those mappings, and extend words to new category members, 
using a combination of preferential looking and referent selection tasks. It also contrasted the word learning skills of children with DS and typical development (TD). Consistent with previous studies (Sakhon et al., 2018), children with DS correctly identified familiar referents and fastmapped pseudowords to unknown objects by mutual exclusivity. Notably, although our research is not the first to study extension skills in DS (see Mervis \& Bertrand, 1995), it is the first to demonstrate that children with this syndrome can reliably use shape information to extend recently acquired words to objects with the same shape but different color. Importantly, there were no differences in the identification of familiar words, fast mapping, or extension skills between children with DS and their TD mental-age pairs; however, children with DS remember fewer word-object mappings than those with TD.

Our work also contributes to understanding linguistic development in DS by examining the interactions between word learning ability and the learning context and cognitive and linguistic skills of children with DS. We show that mental age, verbal skills, and the perceptual features of newly learned objects relate to word learning skills, particularly retention ability, but not to extension skills. More importantly, there were no differences in the relationship between these skills between children with DS and TD. Together, these findings provide evidence about word learning mechanisms in children with DS and offer clues about factors involved in extending and retaining new labels during childhood.

\section{Fast-Mapping Skills in Children with DS}

In this study, children with DS with a mental age of 3 years demonstrated a capacity to use strategies for word learning equivalent to that of their peers with TD. They showed an ability 
to fast-map words; that is, they associated a pseudoword with a novel object. The fast-mapping ability of children with DS has been reported in less perceptually challenging scenarios using two or three competitor items (Bird et al., 2004; Chapman et al., 1990, 2006; McDuffie et al., 2007; Sakhon et al., 2018). Except for McDuffie et al. (2007) and Sakhon et al. (2018), these studies presented the pseudowords in the context of explicit naming. In our study, however, participants had to infer the referent of the novel word by excluding three distractors with already-known names, and extend the word based on the shape of the novel object. Since the novel object was presented among three other familiar objects, the disambiguation phase relied on the mutual exclusivity principle. This kind of presentation combines the well-controlled settings of an experimental task with a learning environment akin to those that children encounter in normal settings, where various objects can compete simultaneously and it is necessary to infer the referent of words based on external cues (Clerkin et al., 2017).

Both the present and previous studies (e.g., Chapman et al., 1990; Chapman et al., 2006) have evaluated children with DS in a wide range of mental ages, which complicates determining the age at which this ability emerges. However, fast-mapping skills may have a similar developmental trajectory in children with and without DS, particularly as the two groups show a similar word learning rate when controlling for mental age (Galeote et al., 2011; Mason-Apps et al., 2020). Given the pivotal role in word learning of correctly inferring the referent of a novel word, future studies should examine the specific age at which this ability first appears in children with DS, and its developmental trajectory.

\section{Extension Skills in Children with DS}

In the extension phase, children with and without DS looked significantly more at the target object when the trained label was presented, demonstrating that they can use shape 
information to extend the label to members of the same category. Mervis and Bertrand (1995) evaluated extension skills in younger children with DS (with a chronological age of 2-3 years). They found that only $40 \%$ of children with DS with high vocabulary levels could extend labels to new examples of the same category. The authors argued that the extension skills in DS are probably related to the vocabulary spurt. In our study, with an average mental age of 3.44 years and an average vocabulary of 367 words, participants with DS have already experienced the vocabulary spurt (see Galeote et al., 2011, for normative data), but only $65 \%$ of these successfully achieved our extension task. Note that all of the variables we measure to explore individual differences are unrelated to extension skills. The question thus remains as to why some children failed to extend words.

A first hypothesis is that participants with DS who failed to extend the labels needed more naming exposures to use the shape of the referent to extend new labels. Previous research using computational models to explore neural-like learning mechanisms in people with DS has shown evidence of slow learning patterns associated with atypical long-term potentiation and an enhanced long-term depression mechanism (Tovar et al., 2018). Although past research has shown that children with DS do not benefit from a larger number of exposures in fast-mapping tasks (Chapman et al., 2006), it is still not known whether it benefits extension skills. Repetition could prevent under- or overextensions. Our results also show that extension and retention skills are not driven by the same variables, and are probably independent processes, since there are words that do not belong to larger categories.

A second hypothesis posits an attentional bias caused by introducing novel information (Houston-Price \& Nakai, 2004). In our task, this bias could be caused by the introduction of three unseen objects in the extension phase; these objects may have attracted the attention of 
children with DS, causing some of them to fail to extend the learned label. This hypothesis is based on the observation that children with DS, regardless of the linguistic stimulus, looked less at the target in the extension phase than children with TD. Thus, although children with DS can generalize words, some of them may struggle to pay attention to the target in complex environments, since they require more effort to maintain the same level of attention as their mental-age pairs with TD (Angulo-Chavira et al., 2017; Díaz \& Zurron, 1995; Kakigi et al., 1994; Kaneko et al., 1996; Seidl et al., 1997).

\section{Retention Skills in Children with DS}

Our results showed that children with DS remember fewer novel words for at least five minutes than their TD peers (approximately three and four words of a total of eight, respectively). This result should be seen against a background of mixed results. Ashworth et al. (2017) reported that participants with DS were able to retain fewer items than controls with TD. However, other studies have found no differences in retention. Bird et al. (2004) reported that individuals both with DS and with TD remembered only one or two out of eight words, and Chapman et al. (1990) found that the percentage of retention was slightly lower (but not significant difference) for the participants with DS than their mental age-matched TD controls ( $77 \%$ and $81 \%$, respectively). Similar results were found in a more recent study by Sakhon et al. (2018), where participants with and without DS remembered two to three words out of four; as in the present study, they examined retention capacity five minutes after exposure to novel wordobject pairs.

The disparity in these results may stem from the specific properties of the task in each case. Ashworth et al. (2017) used twelve novel words, and although they used novel labels for animal names to make the task attractive to children, the large number of object-label pairs made 
the task more challenging for children with DS. Bird et al. (2004) assessed retention through the retelling of the story; however, assessing retention through production makes the task highly challenging for both groups, as seen in the number of items they remembered. In Chapman et al. (1990) the stimuli were physical objects, which gives the participants more perceptual cues, making their task less demanding than ours. As in our study, Sakhon et al. (2018) used digital images, but whereas we presented four images, they presented three, a smaller number of competitors for the novel label-object pair that made the task less challenging for those with DS. It is possible that reducing the cognitive demand of the task may help children with DS attain the retention skills observed in children with TD.

It is worth considering whether the specific characteristics and demands of the task (e.g., the number of objects and learned labels) intervene in the fast-mapping outcomes. In this regard, it is important to consider additional variables that may affect word learning through a few exposures.

\section{Mental Age Modulates Word Retention in DS}

We explored whether mental age, chronological age, or karyotype explain extension and retention skills. Although none of these variables were found to relate to extension skills, mental age was the best predictor of retention of learned words. In addition, the influence of mental age in retention was similar in children with and without DS: retention skills improve with an increase in mental age.

Since the behavioral phenotype of children with DS involves different levels of intellectual disabilities from mild to severe (Chapman \& Hesketh, 2000), it is unsurprising that chronological age does not explain the outcome of the retention task. This difference between mental and chronological age depends on multiple factors, such as genetics, environment, 
cognitive stimulation, and social support. Thus, vocabulary acquisition is better explained by mental than chronological age (Galeote et al., 2011).

Karyotype is usually related to general cognitive skills in children with DS. Those with mosaicism tend to have better general cognitive functioning than those with regular trisomy. In fact, the number of trisomy cells correlates negatively with cognitive performance (for a review see Papavassiliou et al., 2015). In the present study, we did not find a relationship between karyotype and retention skills; however, our sample consisted of 21 participants with regular trisomy, 4 with mosaicism, and another 5 unspecified. Thus, it is possible that this analysis was underpowered, and this result should be considered with caution.

\section{Verbal but Not Nonverbal Skills Relate to Word Retention in Children with DS and TD}

Previous studies have found that children with and without DS take advantage of different verbal and nonverbal processes to learn words. One study has found that while syntactic complexity is related to better word learning skills in people with TD, syntactic complexity and mental age were related to word learning in DS (Bird et al., 2004). To explore this idea, we split the mental age test into verbal and nonverbal components. We found that the retention of new labels in both groups of children was related only to verbal skills. A possible explanation is that our particular nonverbal measures (solving block designs and puzzles) are not related to word learning skills. In contrast to verbal measures, which typically involve receptive and expressive vocabulary tasks, nonverbal skills are measured by various means such as visual recognition, puzzles, and matrix reasoning. Previous studies have also reported that children with and without DS benefit from specific linguistic skills, such as syntactic complexity (Bird et al., 2004; 
detailed evaluation is necessary to better understand the contributions of verbal and nonverbal skills in retention of new words.

\section{Perceptual Information about an Object in the Learning Environment Predicts Word}

\section{Learning}

We also explored how dissimilarity in perceptual features between the target object and the distractors in the preferential looking task affected extension and word retention in children with DS and TD. Our results indicated that while perceptual dissimilarity does not affect extension skills, it does impair retention skills of children both with DS and with TD. In particular, when the familiar object presented during the identification phase showed greater

perceptual dissimilarity, children had difficulty remembering the pseudoword presented with that object. These results are in line with theories of word learning that suggest that attention to the novel object is necessary for word learning (Yu \& Smith, 2012), and with recent findings that decreasing the cognitive complexity of a word learning task by reducing color information or the number of distractors improves retention (Horst et al., 2020; Horst \& Simmering, 2015). In the current study, naming a highly dissimilar familiar object may have caused an attentional bias towards that object, decreasing the attentional resources available for the new word-object mappings.

These results are in stark contrast with those of Barrón-Martínez and Arias-Trejo (2020), who found that children with TD and DS could not identify the object corresponding to a familiar word (e.g., arrow) when they had previously heard a word that referred to an object with a similar shape (e.g., pencil). However, a possible explanation for this discrepancy is that the effects of perceptual information are different in word priming than in word learning. 
Overall, our computer algorithm revealed that perceptual information and competition between target objects and distractors play a critical role in word learning processes. We suggest the use of information-based measures like the Kullback-Leibler divergence (Kullback \& Leibler, 1951) to measure and control perceptual competition in future studies regarding word learning.

\section{Considerations and Future Directions}

Although the current study provides solid evidence identifying the word learning mechanisms that are preserved in children with DS, our results should be interpreted considering the following limitations. First, the global COVID-19 pandemic interrupted data collection. Our sample size was smaller than initially planned, and we were prevented from matching children with DS to children with TD based on mental age (but see the Supplementary Material 1). Second, past studies with children with DS have included participants with large age ranges (e.g., 11 to 26 years). Here, we restricted the chronological age of our participants to a range of 6 to 13 years. However, even within this controlled age range, participants' linguistic performance might still differ because of differences in life experience and language exposure.

Differences in the color and shape features of the competitors were quantified using the Kullback-Leibler divergence. Although this method is objective in terms of the bottom-up information provided by the environment, it could differ from subjective judgments of similarity: two objects considered dissimilar by the algorithm could appear similar to a subject.

Nevertheless, both our study and a prior one (Horst et al., 2020) have provided evidence that perceptual features of objects modulated word learning. It is still an open question how perception and bottom-up information interact in word learning. Future studies should explore 
the functional relationship between subjective perception and perceptual information from the environment.

Our results also showed that the disambiguation and extension skills of children with DS are comparable to those of children with TD. However, in our design, learning the pseudowords required only lexical information. It also remains unresolved whether people with DS may have difficulty using word learning mechanisms that tap into other linguistic domains, such as morphology. For example, people with DS experience particular challenges producing morphemes (Galeote et al., 2014), but the comprehension of some morphemes is preserved (Abreu-Mendoza et al., 2020). Future studies should also investigate whether children with DS, despite their impairments in production and working memory, employ morphological information to bootstrap the referent of new words, as do children with TD (Jolly \& Plunkett, 2008). Finally, even though our results are consistent with those of studies of English-speaking children with DS, it remains unclear how cross-linguistic differences may affect their word learning skills. Studies examining such differences in children with TD have found that young learners of English and Spanish with the same vocabulary skills differ in their wordgeneralization skills (Hahn \& Cantrell, 2012); however, studies of children in a similar age range as our participants have not observed such differences (Gathercole \& Min, 1997).

\section{Conclusions}

Although children with DS understand a similar number of words as their TD peers during the first years of life, there is a gap in our knowledge about the mechanisms that support word learning in children with DS. Our study found that children with DS have similar abilities to identify familiar objects and to map and extend novel word-object associations, but weaker word retention skills than those with TD. We also found that individual differences in retention 
skills of both children with DS and TD are positively related to vocabulary, general cognitive skills, and perceptual differences between the target and non-target objects. These results offer a comprehensive examination of the word learning mechanism in children with DS. 


\section{References}

Abbeduto, L., Warren, S. F., \& Conners, F. A. (2007). Language development in Down syndrome: From the prelinguistic period to the acquisition of literacy. Mental Retardation and Developmental Disabilities Research Reviews, 13(3), 247-261. https://doi.org/10.1002/mrdd.20158

Abreu-Mendoza, R. A., Jasso, T., Soto-Alba, E. E., \& Arias-Trejo, N. (2020). Receptive number morphosyntax in children with Down syndrome. Language and Cognition, 12(4), 679-704. https://doi.org/10.1017/langcog.2020.18

Angulo-Chavira, A. Q., García, O., \& Arias-Trejo, N. (2017). Pupil response and attention skills in Down syndrome. Research in Developmental Disabilities, 70, 40-49. http://www.embase.com/search/results?subaction=viewrecord\&from=export\&id=L6181238 45\%0Ahttp://dx.doi.org/10.1016/j.ridd.2017.08.011

Arias-Trejo, N. (2010). Young children's extension of novel labels to novel animate items in three testing conditions. International Journal of Behavioral Development, 34(3), 206-217. http://proxy.libraries.smu.edu/login?url=http://search.ebscohost.com/login.aspx?direct=true $\& \mathrm{db}=$ eue $\& A N=49714561 \&$ site $=$ ehost-live \&scope $=$ site\%0A10.1177/0165025409350951

Ashworth, A., Hill, C. M., Karmiloff-Smith, A., \& Dimitriou, D. (2017). A cross-syndrome study of the differential effects of sleep on declarative memory consolidation in children with neurodevelopmental disorders. Developmental Science, 20(2). https://doi.org/10.1111/desc. 12383

Baayen, R. H., Davidson, D. J., \& Bates, D. M. (2008). Mixed-effects modeling with crossed random effects for subjects and items. Journal of Memory and Language, 59, 390-412. https://ac.els-cdn.com/S0749596X07001398/1-s2.0-S0749596X07001398- 
main.pdf?_tid=3e2aa272-da0f-4c63-ad5f-

7cef039faa25\&acdnat=1533815932_4731183dc2fe380f5a6142be28fc3c43

Barr, D. J. (2008). Analyzing "visual world" eyetracking data using multilevel logistic regression. Journal of Memory and Language, 59(4), 457-474. https://doi.org/10.1016/j.jml.2007.09.002

Barrón-Martínez, J. B., \& Arias-Trejo, N. (2020). Perceptual similarity effect in people with Down syndrome. International Journal of Developmental Disabilities. https://doi.org/10.1080/20473869.2020.1729016

Bates, D., Maechler, M., Bolker, B., \& Walker, S. (2015). Fitting Linear Mixed-Effects Models Using lme4. Journal of Statistical Software, 67(1), 1-48.

Bion, R. A. H., Brovsky, A., \& Fernald, A. (2013). Fast mapping, slow learning: Disambiguation of novel word-object mapping in relation to vocabulary learning at 18,24 , and 30 months. Cognition, 126, 39-53.

Bird, E. K. R., Chapman, R. S., \& Schwartz, S. E. (2004). Fast mapping of words and story recall by individuals with Down syndrome. Journal of Speech, Language, and Hearing Research, 47(6), 1286-1300. https://doi.org/10.1044/1092-4388(2004/097)

Bird, E. K. R., Gaskell, A., Babineau, M. D., \& MacDonald, S. (2000). Novel word acquisition in children with Down syndrome: Does modality make a difference? Journal of Communication Disorders, 33(3), 241-266. https://doi.org/10.1016/S0021-9924(00)00022-8

Canfield, R. L., Smith, E. G., Brezsnyak, M. P., Snow, K. L., Aslin, R. N., Haith, M. M., Wass, T. S., \& Adler, S. A. (1997). Information Processing Through the First Year of Life: A Longitudinal Study Using the Visual Expectation Paradigm. Monographs of the Society for Research in Child Development, 62(2), i. https://doi.org/10.2307/1166196 
Carey, S., \& Barlett, E. (1978). Acquiring a single new word. In Proceedings of the Stanford Child Language Conference (Vol. 15, pp. 17-29).

Chambers, J. M. (1992). Linear Models. In J. M. Chambers \& T. J. Hastie (Eds.), Statistical Models in S. Wadsworth \& Brooks/Cole. https://doi.org/10.2307/2533532

Chapman, R. S., Bird, E. K. R., \& Schwartz, S. E. (1990). Fast mapping of words in event contexts by children with Down syndrome. Journal of Speech and Hearing Disorders, 55(4), 761770. https://doi.org/10.1044/jshd.5504.761

Chapman, R. S., \& Hesketh, L. J. (2000). Behavioral phenotype on individuals with Down Syndrome. Mental Retardation and Developmental Disabilities Research Reviews, 6(2), 8495.

Chapman, R. S., Sindberg, H., Bridge, C., Gigstead, K., \& Hesketh, L. (2006). Effect of memory support and elicited production on fast mapping of new words by adolescents with Down syndrome. Journal of Speech, Language, and Hearing Research, 49(1), 3-15. https://doi.org/10.1044/1092-4388(2006/001)

Checa, E., Galeote, M., \& Soto, P. (2016). The composition of early vocabulary in Spanish children with Down syndrome and their peers with typical development. American Journal of SpeechLanguage Pathology, 25(4), 605-619. https://doi.org/10.1044/2016_AJSLP-15-0095

Clerkin, E. M., Hart, E., Rehg, J. M., Yu, C., \& Smith, L. B. (2017). Real-world visual statistics and infants' first-learned object names. Philosophical Transactions of the Royal Society B: Biological Sciences, 372(1711). https://doi.org/10.1098/rstb.2016.0055

Delle Luche, C., Durrant, S., Poltrock, S., \& Floccia, C. (2015). A methodological investigation of the Intermodal Preferential Looking paradigm: Methods of analyses, picture selection and data rejection criteria. Infant Behavior and Development, 40, 151-172. 
https://doi.org/10.1016/j.infbeh.2015.05.005

Díaz, F., \& Zurron, M. (1995). Auditory evoked potentials in Down's syndrome. Electroencephalography and Clinical Neurophysiology/Evoked Potentials, 96(6), 526-537. https://doi.org/10.1016/0013-4694(95)00080-I

Faul, F., Erdfelder, E., Buchner, A., \& Lang, A. G. (2009). Statistical power analyses using G*Power 3.1: Tests for correlation and regression analyses. Behavior Research Methods, 41(4), 1149-1160. https://doi.org/10.3758/BRM.41.4.1149

Fernald, A. (1985). Four-month-old infants prefer to listen to motherese. Infant Behavior and Development, 8(2), 181-195. https://doi.org/10.1016/S0163-6383(85)80005-9

Frank, M. C., Braginsky, M., Yurovsky, D., \& Marchman, V. A. (2017). Wordbank: An open repository for developmental vocabulary data. Journal of Child Language, 44(3), 677-694. https://doi.org/10.1017/S0305000916000209

Galeote, M., Sebastián, E., Checa, E., Rey, R., \& Soto, P. (2011). The development of vocabulary in Spanish children with Down syndrome: Comprehension, production, and gestures. Journal of Intellecutal \& Developmental Disability, 36(3), 184-196.

Galeote, M., Soto, P., Lamela, E., Checa, E., Pulido, L., \& Rey, R. (2006). Adaptación de los Inventarios de Desarrollo Comunicativo MacArthur-Bates para el estudio del desarrollo del lenguaje en niños con síndrome de Down. Revista de Atención Temprana, 9, 4-16.

Galeote, M., Soto, P., Sebastián, E., Checa, E., \& Sánchez-Palacios, C. (2014). Early grammatical development in Spanish children with Down syndrome. Journal of Child Language, 41(1), 111-131. https://doi.org/10.1017/S0305000912000591

Gathercole, V. C. M., \& Min, H. (1997). Word meaning biases or language-specific effects? Evidence from English, Spanish and Korean. First Language, 17(49), 031-056. 
https://doi.org/10.1177/014272379701704902

Gelman, S. A., Croft, W., Fu, P., Clausner, T., \& Gottfried, G. (1998). Why is a pomegranate an apple? The role of shape, taxonomic relatedness, and prior lexical knowledge in children's overextensions of apple and dog. Journal of Child Language, 25(2), 267-291. https://doi.org/10.1017/S0305000998003420

Gibson, J. J. (1958). Visually Controlled Locomotion and Visual Orientation in Animals. British Journal of Psychology, 49(3), 182-194. https://doi.org/10.1111/j.2044-8295.1958.tb00656.x

Gleitman, L. (1990). The Structural Sources of Verb Meanings. Language Acquisition, 1(1), 3-55. https://doi.org/10.1207/s15327817la0101_2

Golinkoff, R. M., Hirsh-Pasek, K., Bailey, L. M., \& Wenger, N. R. (1992). Young children and adults use lexical principles to learn new nouns. Developmental Psychology, 28(1), 99-108. https://doi.org/10.1037//0012-1649.28.1.99

Goncalves, A., Abrantes, J., Saponaro, G., Jamone, L., \& Bernardino, A. (2014). Learning intermediate object affordances: Towards the development of a tool concept. IEEE ICDLEPIROB 2014 - 4th Joint IEEE International Conference on Development and Learning and on Epigenetic Robotics, 482-488. https://doi.org/10.1109/DEVLRN.2014.6983027

Hahn, E. R., \& Cantrell, L. (2012). The shape-bias in Spanish-speaking children and its relationship to vocabulary. Journal of Child Language, 39(2), 443-455. https://doi.org/10.1017/S030500091100016X

Halberda, J. (2003). The development of a word-learning strategy. Cognition, 87, B23-B34.

Hartley, C., Bird, L. A., \& Monaghan, P. (2019). Investigating the relationship between fast mapping, retention, and generalisation of words in children with autism spectrum disorder and typical development. Cognition, $187, \quad 126-138$. 
https://doi.org/10.1016/j.cognition.2019.03.001

Holler, D. E., Fabbri, S., \& Snow, J. C. (2020). Object responses are highly malleable, rather than invariant, with changes in object appearance. Scientific Reports, 10(1), 1-14. https://doi.org/10.1038/s41598-020-61447-8

Horst, J. S., \& Hout, M. C. (2016). The Novel Object and Unusual Name (NOUN) Database: A collection of novel images for use in experimental research. Behavior Research Methods, 48(4), 1393-1409. https://doi.org/10.3758/s13428-015-0647-3

Horst, J. S., \& Samuelson, L. K. (2008). Fast mapping but poor retention by 24-month-old infants. Infancy, 13(2), 128-157. https://doi.org/10.1080/15250000701795598

Horst, J. S., Scott, E. J., \& Pollard, J. A. (2010). The role of competition in word learning via referent selection. Developmental Science, 13(5), 706-713. https://doi.org/10.1111/j.14677687.2009.00926.x

Horst, J. S., \& Simmering, V. R. (2015). Category learning in a dynamic world. Frontiers in Psychology, 6(JAN). https://doi.org/10.3389/fpsyg.2015.00046

Horst, J. S., Twomey, K. E., Morse, A. F., Nurse, R., \& Cangelosi, A. (2020). When Object Color Is a Red Herring: Extraneous Perceptual Information Hinders Word Learning via Referent Selection. IEEE Transactions on Cognitive and Developmental Systems, 12(2), 222-231. https://doi.org/10.1109/TCDS.2019.2894507

Houston-Price, C., \& Nakai, S. (2004). Distinguishing novelty and familiarity effects in infant preference procedures. Infant and Child Development, 13(4), 341-348. https://doi.org/10.1002/icd.364

Houston-Price, C., Plunkett, K., \& Duffy, H. (2006). The use of social and salience cues in early word learning. Journal of Experimental Child Psychology, 95(1), 27-55. 
https://doi.org/10.1016/j.jecp.2006.03.006

Huettig, F., Olivers, C. N. L., \& Harsuiker, R. J. (2011). Looking, language, and memory: Bridging research from the visual world and vidual paradigms. Acta Pyschologica, 137, 138-150.

Jackson-Maldonado, D., Thal, D., Marchman, V., Bates, E., \& Gutierrez-Clellen, V. (1993). Early lexical development in Spanish-speaking infants and toddlers. Journal of Child Language, $20,523-549$.

Jackson-Maldonado, D., Thal, D., Marchman, V., Newton, T., Fenson, L., \& Conboy, B. (2003). MacArthur inventarios del desarrollo de habilidades comunicativas. User's guide and technical manual. Brookes.

Jarrold, C., Thorn, A. S. C., \& Stephens, E. (2009). The relationships among verbal short-term memory, phonological awareness, and new word learning: Evidence from typical development and Down syndrome. Journal of Experimental Child Psychology, 102(2), 196218. https://doi.org/10.1016/j.jecp.2008.07.001

Jolly, H., \& Plunkett, K. (2008). Inflectional Bootstrapping in 2-year-olds. Language and Speech, 51, 45-59. https://doi.org/10.1177/00238309080510010401

Justicia, F. (1995). El desarrollo del vocabulario. Diccionario de frecuencias. Servicio de Publicaciones. Universidad de Granada.

Kakigi, R., Neshige, R., Matsuda, Y., \& Kuroda, Y. (1994). Do patients with Down's syndrome recognize Mickey Mouse? Journal of the Neurological Sciences, 121(1), 22-26. https://doi.org/10.1016/0022-510X(94)90151-1

Kaneko, W. M., Phillips, E. L., Riley, E. P., \& Ehlers, C. L. (1996). EEG findings in fetal alcohol syndrome and Down syndrome children. Electroencephalography and Clinical Neurophysiology, 98(1), 20-28. https://doi.org/10.1016/0013-4694(95)00189-1 
Kullback, S., \& Leibler, R. A. (1951). on Information and Sufficiency. The Annals of Mathematical Statistics, 79-86. https://www.jstor.org/stable/pdf/2236703.pdf

Landau, B., Smith, L. B., \& Jones, S. S. (1988). The importance of shape in early lexical learning. Cognitive Development, 3(3), 299-321. https://doi.org/10.1016/0885-2014(88)90014-7

Lewis, M., Cristiano, V., Lake, B. M., Kwan, T., \& Frank, M. C. (2020). The role of developmental change and linguistic experience in the mutual exclusivity effect. Cognition, 198. https://doi.org/10.1016/j.cognition.2020.104191

Markman, E. (1990). Constraints children place on word meanings. Cognitive Science, 14, 154173.

Markson, L., \& Bloom, P. (1997). Evidence against a dedicated system for word learning in children. Nature, 385(6619), 813-815. https://doi.org/10.1038/385813a0

Mason-Apps, E., Stojanovik, V., Houston-Price, C., Seager, E., \& Buckley, S. (2020). Do Infants With Down Syndrome Show an Early Receptive Language Advantage? Journal of Speech, Language, and Hearing Research: JSLHR, 63(2), 585-598. https://doi.org/10.1044/2019_JSLHR-19-00157

McDuffie, A. S., Sindberg, H. A., Hesketh, L. J., \& Chapman, R. S. (2007). Use of speaker intent and grammatical cues in fast-mapping by adolescents with Down syndrome. Journal of Speech, Language, and Hearing Research, 50(6), 1546-1561. https://doi.org/10.1044/1092$4388(2007 / 105)$

Mervis, C. B., \& Bertrand, J. (1995). Acquisition of the novel name-nameless category (N3C) principle by young children who have Down syndrome. American Journal on Mental Retardation, 100(3), 231-243.

Mosse, E. K., \& Jarrold, C. (2011). Evidence for preserved novel word learning in Down syndrome 
suggests multiple routes to vocabulary acquisition. Journal of Speech, Language, and Hearing Research, 54(4), 1137-1152. https://doi.org/10.1044/1092-4388(2010/09-0244)

Nelson, K. (1988). Constraints on word learning? Cognitive Development, 3(3), 221-246. https://doi.org/10.1016/0885-2014(88)90010-X

Papavassiliou, P., Charalsawadi, C., Rafferty, K., \& Jackson-Cook, C. (2015). Mosaicism for trisomy 21: A review. American Journal of Medical Genetics, Part A, 167(1), 26-39. https://doi.org/10.1002/ajmg.a.36861

Perry, L. K., Axelsson, E. L., \& Horst, J. S. (2016). Learning What to Remember: Vocabulary Knowledge and Children's Memory for Object Names and Features. Infant and Child Development, 25(4), 247-258. https://doi.org/10.1002/icd.1933

Quilis, A. (1983). Frecuencia de los esquemas acentuales en español. In Estudios ofrecidos a Emilio Alarcos Llorach (con motivo de sus XXV años de docencia en la Universidad de Oviedo) (pp. 113-126).

Quine, W. V. O. (1960). Word and Object. In The MIT Press (Ed.), Word and Object. https://doi.org/10.7551/mitpress/9636.001.0001

R Core Team. (2019). $R$ : A language and environment for statistical computing, reference index version 3.5.3.

Real Academia Española. (2018). Diccionario de la lengua espanola.

Rujas, I., Casla, M., Mariscal, S., Lázaro López-Villaseñor, M., \& Murillo Sanz, E. (2019). Effects of grammatical category and morphology on fast mapping in typically developing and late talking toddlers. First $\quad$ Language, $39(3), \quad$ 249-280. https://doi.org/10.1177/0142723719828258

Sakhon, S., Edwards, K., Luongo, A., Murphy, M., \& Edgin, J. (2018). Small Sets of Novel Words 
Are Fully Retained after 1-Week in Typically Developing Children and Down Syndrome: A Fast Mapping Study. Journal of the International Neuropsychological Society, 24(9), 955965. https://doi.org/10.1017/S1355617718000450

Samuelson, L. K., \& Smith, L. B. (1999). Early noun vocabularies: Do ontology, category structure and syntax correspond? Cognition, 73(1), 1-33. https://doi.org/10.1016/S00100277(99)00034-7

Sattler, J. (2010). Evaluación infantil: Fundamentos cognitivos: Vol. I (5ta ed.). Manual Moderno.

Seidl, R., Hauser, E., Bernert, G., Marx, M., Freilinger, M., \& Lubec, G. (1997). Auditory evoked potentials in young patients with Down syndrome. Event- related potentials (P3) and histaminergic system. Cognitive Brain Research, 5(4), 301-309. https://doi.org/10.1016/S0926-6410(97)00008-6

Silverman, W. (2007). Down syndrome: Cognitive phenotype. Mental Retardation and Developmental Disabilities Research Reviews, 13(3), 228-236. https://doi.org/10.1002/mrdd.20156

Smith, L. B. (1995). Self-organizing processes in learning to learn words: Development is not induction. In Basic and applied perspectives on learning, cognition, and development (pp. 132).

Smith, L. B., Colunga, E., \& Yoshida, H. (2010). Knowledge as Process: Contextually Cued Attention and Early Word Learning. Cognitive Science, 34(7), 1287-1314. https://doi.org/10.1111/j.1551-6709.2010.01130.x

Smith, L. B., Jones, S. S., Landau, B., Gershkoff-Stowe, L., \& Samuelson, L. (2002). Object name learning provides on-the-job training for attention. Psychological Science, 13(1), 13-19. https://doi.org/10.1111/1467-9280.00403 
Spiegel, C., \& Halberda, J. (2011). Rapid fast-mapping abilities in 2-year-olds. Journal of Experimental Child Psychology, 109, 132-140.

Swanson, W. H., \& Cohen, J. M. (2003). Color vision. Ophthalmology Clinics, 16(2), 179-203. https://doi.org/10.1016/S0896-1549(03)00004-X

Tovar, Á. E., Westermann, G., \& Torres, A. (2018). From altered synaptic plasticity to atypical learning: A computational model of Down syndrome. Cognition, 171, 15-24. https://doi.org/10.1016/j.cognition.2017.10.021

Virtanen, P., Gommers, R., Oliphant, T. E., Haberland, M., Reddy, T., Cournapeau, D., Burovski, E., Peterson, P., Weckesser, W., Bright, J., van der Walt, S. J., Brett, M., Wilson, J., Millman, K. J., Mayorov, N., Nelson, A. R. J., Jones, E., Kern, R., Larson, E., ... Vázquez-Baeza, Y. (2020). SciPy 1.0: fundamental algorithms for scientific computing in Python. Nature Methods, 17(3), 261-272. https://doi.org/10.1038/s41592-019-0686-2

Wechsler, D. (2011). Escala Wechsler de Inteligencia para los niveles preescolar y primario-III. Manual Moderno.

Yu, C., \& Smith, L. B. (2012). Embodied attention and word learning by toddlers. Cognition, 125(2), 244-262. https://doi.org/10.1016/j.cognition.2012.06.016 